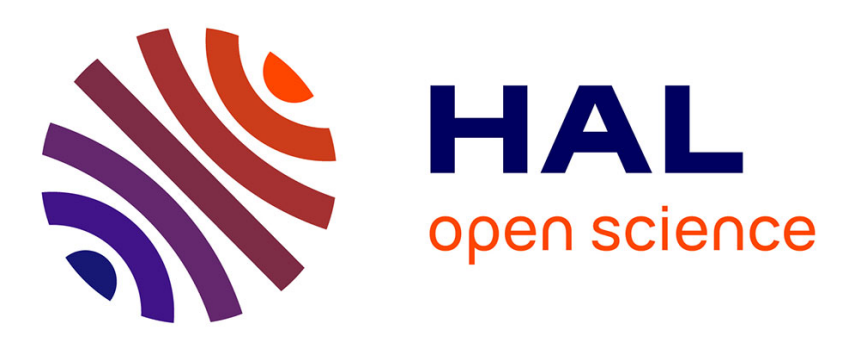

\title{
Assessment of an anisotropic coarse-grained model for cis -1,4-polybutadiene: a bottom-up approach
}

Ioannis Tanis, Bernard Rousseau, Laurent Soulard, Claire Lemarchand

\section{To cite this version:}

Ioannis Tanis, Bernard Rousseau, Laurent Soulard, Claire Lemarchand. Assessment of an anisotropic coarse-grained model for cis -1,4-polybutadiene: a bottom-up approach. Soft Matter, 2020, 17 (3), pp.621-636. 10.1039/d0sm01572e. hal-03035892

\section{HAL Id: hal-03035892 \\ https://hal.science/hal-03035892}

Submitted on 16 Nov 2021

HAL is a multi-disciplinary open access archive for the deposit and dissemination of scientific research documents, whether they are published or not. The documents may come from teaching and research institutions in France or abroad, or from public or private research centers.
L'archive ouverte pluridisciplinaire HAL, est destinée au dépôt et à la diffusion de documents scientifiques de niveau recherche, publiés ou non, émanant des établissements d'enseignement et de recherche français ou étrangers, des laboratoires publics ou privés. 


\title{
Soft Matter
}

ARTICLE TYPE

Cite this: DOI: $00.0000 / x x x x x x x x x x$

\section{Assessment of an anisotropic coarse-grained model for cis-1,4-polybutadiene: a bottom-up approach ${ }^{\dagger}$}

\author{
Ioannis Tanis, ${ }^{a}$ Bernard Rousseau, ${ }^{b}$ Laurent Soulard,,${ }^{a, c}$ and Claire A. Lemarchand, ${ }^{* a, c}$
}

Received Date

Accepted Date

DOI: $00.0000 / x x x x x x x x x x$

\begin{abstract}
The spherical representation usually utilized for the coarse-grained particles of soft matter systems is an assumption and pertinent studies have shown that both structural and dynamical properties can depend on anisotropic effects. On these grounds, we develop coarse-grained equations of motion which take into account explicitly the anisotropy of the beads. As a first step, this model incorporates only conservative terms. Inclusion of the dissipative and random terms is in principle possible but is beyond the scope of this study. The translational dynamics of the beads is tracked using the position and momentum of their center of mass, while their rotational dynamics is modeled by representing their orientation through the use of quaternions, similarly to the case of rigid bodies. The associated force and torque controlling the motion are derived from atomistic molecular dynamics (MD) simulations via a bottom-up approach and define a coarse-grained potential. The assumptions of the model are clearly stated and checked for a reference system of a cis-1,4-polybutadiene melt. In particular, the choice of the angular velocity as a slow variable is justified by comparing its dynamics to atomic vibrations. The accuracy of this approach to reproduce static structural features of the polymer melt is assessed.
\end{abstract}

\section{Introduction}

Coarse-grained (CG) simulations of simple liquids, polymers and biomolecular systems are a powerful tool to obtain structural and even dynamical properties more efficiently than full atomistic simulations. This is due to the fact that CG simulations reduce the number of degrees of freedom to be considered and allow for a larger time step to be used $1+3$. Among the coarse-graining strategies, bottom-up approaches are appealing because they root the whole coarse-graining framework on the more reliable atomistic simulations explicitly. The derivation of the full coarse-grained equations of motion from the atomistic equations of motion has been the subject of a detailed study ${ }^{4}$ and relies on a good time scale separation between slow CG variables and fast atomic vibrations. The full coarse-grained equations involve a conservative force which is exactly the force on a bead averaged over the atomic degrees of freedom and which depend in principle on all the CG degrees of freedom 24 . This many-body potential is not easy to compute and many approximations need to be made to

\footnotetext{
${ }^{a}$ CEA, DAM, DIF, 91297 Arpajon Cedex, France.

${ }^{b}$ Université Paris-Saclay, CNRS, Institut de Chimie Physique UMR 8000, 91405 Orsay, France.

${ }^{c}$ Université Paris-Saclay, CEA, Laboratoire Matière sous Conditions Extrêmes, 91680, Bruyères-le-Chatel, France.

* E-mail: claire.lemarchand@cea.fr

$\dagger$ Electronic Supplementary Information (ESI) available: [details of any supplementary information available should be included here]. See DOI: $10.1039 / \mathrm{cXsm00000x/}$
}

obtain a CG potential tractable to calculate ${ }^{2}$. Different sets of approximations exist but none of them is able to satisfy at the same time the following features: accuracy on different structural properties, efficiency and transferability 2 . Different bottom-up approaches are utilized to evaluate the CG potential, such as the iterative Boltzmann technique $\mathrm{e}^{56}$, the force matching method 7.49 , and the more recent trajectory mapping method 10 . In this work, we chose the force matching method also used in Ref. 4 in its derivation of the full CG equations of motion, and for which the approximations are clearly stated. The full CG equations of motion also contain dissipative and random forces which can be obtained from a bottom-up approach. The friction coefficient of the dissipative forces can be evaluated in constrained atomic simulations where the CG variables are kept constant and only the atomic degrees of freedom are allowed to relax. The use of this bottom-up friction coefficient allows one to obtain not only structural properties comparable to those obtained in atomistic simulations but also to describe accurately the dynamics 13.

Prior to the establishment of the full CG equations of motion from the atomistic simulations and of the ensuing approximations made to calculate a tractable CG potential and friction coefficient, the CG variables themselves need to be selected ${ }^{2}$. Usually the CG variables are chosen to be the positions and velocities of the bead centers of mass 2 . Nevertheless, this is not always sufficient to fully describe the system both structurally and dynamically 2 . The anisotropy of the bead in particular is an obvious complementary coarse-grained variable when beads are clearly anisotropic, 
like ellipsoidal colloids $\frac{17-20}{2}$. Anisotropic CG potentials have been derived in a bottom-up approach for example for amino acids in proteins ${ }^{21 \mid 22}$ and for monomers in polystyrene 2324 or semiflexible ring polymers $25 \mid 26$. In these studies, the improvement of the structural properties of the CG system when the anisotropy is taken into account has been tested by using CG Monte-Carlo simulations. In cis-1,4-polybutadiene also, the beads, whether they correspond to one or several monomers, are anisotropic 11 and this anisotropy appears to have an important impact on the CG potential ${ }^{16}$ and consequently on the structural properties. Moreover, it has been argued that taking into account the bead orientation as a CG variable can improve the calculation of the dynamical properties in cis-1,4-polybutadiene ${ }^{16}$. Indeed, following the bottom-up approach of Ref. ${ }^{[4}$, Lemarchand and coworkers ${ }^{16}$ evaluated the friction coefficient in constrained simulations where the bead positions and velocities are fixed and noticed that the relaxation of the bead orientation slows down dramatically compared to unconstrained simulations. This implies a coupling between translational and rotational degrees of freedom which cannot be considered as slow independently. It also suggests that the calculation of the friction coefficient in these constrained simulations neglects the effect of the slow relaxation of the bead orientation. The latter can have a strong impact on the dynamical properties.

For the aforementioned reasons, this work tackles the problem of applying the theoretical framework proposed in Ref. 4 to the case where the coarse-grained variables include not only the positions and velocities of the bead centers of mass but also their orientations. As a first step in that direction, this work aims at expressing explicitly the CG bead orientation in terms of atomistic variables, deriving the full atomic equations of motion for the previously defined CG variables, and obtaining the corresponding CG equations of motion including only conservative forces. The derivation of the dissipative and random forces will be the subject of a future study. The earlier mentioned coarse-graining methodology is applied to a cis-1,4-polybutadiene melt, a usual reference system to test coarse-graining procedures 16 27. 30 . The validity of all the approximations made to derive the CG equations of motion is tested on this system. The obtained CG anisotropic model is implemented and the corresponding CG dynamical simulations are performed. The structural properties of the system obtained in atomistic simulations, isotropic CG simulations and anisotropic CG simulations are compared.

The paper is organized as follows. Methods and technical details for atomistic and CG dynamical simulations are given in Sec. 2. Section 3 describes the coarse-graining methodology and presents the anisotropic CG potential. The locally anisotropic structural properties of the melt obtained in atomistic and anisotropic CG simulations as well as the more common radial distribution functions and chains dimensions obtained in atomistic, isotropic CG and anisotropic CG simulations are compared in Sec. 4 Finally, the approximations done in Sec. 3 are carefully tested and discussed in Sec. 5 Section 6 contains a summary and a conclusion.

\section{Methods}

\subsection{Molecular dynamics simulation details}

The molecular dynamics (MD) simulations were conducted using the STAMP code ${ }^{31 / 32}$, a code developed at CEA and parallelized using MPI. Polymer chains are represented by united-atoms (UA) and the system comprises 200 chains of 12 monomers each. An illustration of a chain is displayed in Fig. 1 1 The force field used is that developed by Harmandaris 33 , the latter being based on an earlier model by Smith $34 \sqrt[35]{ }$. Its detailed parameters have been given in 16 . The cutoff distance used for the van der Waals interactions is of $10 \AA$. The simulations can be carried out in the microcanonical (NVE) ensemble, in the canonical ensemble (NVT) with a Nose-Hoover thermostat using a coupling constant of 0.1 ps or in constant pressure-constant temperature (NPT) ensemble using a Nose-Hoover thermostat and barostat ${ }^{36}$ with the same coupling constant for the thermostat and a coupling constant of 2.6 fs for the barostat. Long-range corrections to the energy and the pressure are added using the approximation that the radial distribution functions are equal to unity beyond the cutoff. The integration algorithm is velocity Verlet and the time step is $1 \mathrm{fs}$.

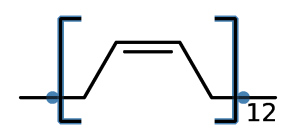

Fig. 1 Illustration of a single chain of cis-1,4-polybutadiene of 12 monomers.

The state point simulated is the same as that chosen in 16 and corresponds to a temperature of $500 \mathrm{~K}$ and ambient pressure. The average density obtained in the MD simulation is $0.772 \mathrm{~g} / \mathrm{cm}^{3}$, while the experimental density of cis-1,4-polybutadiene for high molecular weights of $M_{w}=2-3.10^{5} \mathrm{~kg} / \mathrm{mol}$ is around 0.781 , a difference of about $1 \%$. Furthermore, the density of a melt of cis-1,4-polybutadiene chains of 12 monomers described by a united-atom potential is known to exhibit a difference of only $5 \%$ compared to the density of a melt of very long chains $\frac{38}{38}$. The structural properties of the melt described in this work are consequently expected to be very close to those of a melt with longer chains.

At an initial stage, the melt sample is relaxed for $2 \mathrm{~ns}$ in the NPT ensemble. Then, to obtain the data necessary to construct the coarse-grained potential, simulations are carried out in the NVT ensemble for $10 \mathrm{~ns}$ and the position, velocity and force of each atom is saved every $0.1 \mathrm{ps}$. To obtain the data necessary to calculate autocorrelation functions, simulations are carried out in the NVE ensemble for $1 \mathrm{~ns}$ with a frame-saving period of 0.01ps.

\subsection{Coarse-grained simulations}

The coarse-grained (CG) simulations were also conducted using the STAMP code. The algorithm used to carry out the CG simu- 


\begin{tabular}{lcc}
\hline$\lambda$ & CG iso & CG aniso \\
\hline 1 & total time & \\
2 & 39 & 29 \\
3 & 105 & 71 \\
\hline & 172 & 122 \\
1 & 1.0 & \\
2 & 1.3 & 0.7 \\
3 & 1.4 & 0.9 \\
\hline
\end{tabular}

Table 1 Factor by which the execution time (total time and time per particle and iteration) of the CG simulations is divided compared to an equivalent MD simulation for different levels of coarse-graining $\lambda$

lations is a simple adaptation of an algorithm used to integrate the equations of motion of rigid molecules. The rigid molecule algorithm is that described in Ref. $\frac{39}{39}$ as a modified version of the leap-frog algorithm where an iterative loop on the quaternion at half step is performed to obtain the angular velocity at the next step. It was simply adapted to use the derivative with respect to the distance and the derivative with respect to the angle of a tabulated potential as the force and torque on a bead, respectively. The time step in the CG simulations is of $10 \mathrm{fs}$, ten times larger than that used in the MD simulations. The cutoff distance of the tabulated potential is of $20 \AA$. The simulations are carried out in the NVT ensemble using a Langevin thermostat with a friction coefficient of $10^{12} \mathrm{~s}^{-1}$. An isotropic version of the CG simulations using a potential depending only on the distance and not calculating any torque is also implemented for comparison purposes.

The CG simulations are carried out for the same system at the same state point as that simulated in MD, using initial CG configurations obtained from equilibrated MD configurations. The CG simulations also last 10 ns and the frame-saving period in CG simulations is of $1 \mathrm{ps}$.

\subsection{Comparative performances}

The performances of the MD, isotropic and anisotropic CG simulations are compared on the same system and the same machine for different levels of coarse-graining $\lambda$, number of monomers per bead. The factor by which the total execution time and the time per particle and iteration is divided in CG simulations compared to the corresponding time in MD is listed in Table 1 for all cases. The gain in the total execution time in CG simulations has three origins: (i) the larger time step allows for a brute gain of a factor 10, (ii) the lower number of particles enables a direct gain of $4 \lambda$, (iii) the lower number of particles also leads to a smaller particle density which decreases the execution time per iteration and particle. However, the larger cutoff distance in CG simulation tend to increase the execution time per iteration and particle compared to its MD counterpart. In total, the CG simulations are 1 to 2 orders of magnitude faster than the MD simulations. The anisotropic CG simulations are always a bit slower than the corresponding isotropic simulations, but the gain in speed compared to MD is similar in both cases.

\section{Formulation of the anisotropic model}

3.1 Choice of the slow variables
Taking into account the bead orientation in CG simulations of polymer melts seems necessary for two main reasons, (i) beads can be anisotropic ${ }^{11} 16$ and this can impact the structural properties of the melt and (ii) the dynamics of the bead orientation seem correlated to those of the bead velocity usually chosen as a slow variable $\mathrm{e}^{16}$. Before deriving the CG equations of motion for the bead orientation, we need to verify that it is indeed a slow variable. To do so we compare its characteristic relaxation time to that of the usual slow variables, positions and velocities of the beads' centers of mass, and the usual fast variables, total forces on the beads 4 .

\subsubsection{Bead orientation.}

To quantify the bead orientation, we propose to use the wellknown eigenvectors of the bead inertia tensor, similarly to what is done for rigid bodies. More specifically, the inertia tensor of bead $\mu$ is defined as:

$$
\mathbf{I}_{\mu}=\sum_{i \in \mu} m_{i}\left(\left(\Delta \mathbf{r}_{i} \cdot \Delta \mathbf{r}_{i}\right) \mathbf{E}-\Delta \mathbf{r}_{i} \otimes \Delta \mathbf{r}_{i}\right),
$$

where $m_{i}$ is the mass of atom $i, \Delta \mathbf{r}_{i}=\mathbf{r}_{i}-\mathbf{R}_{\mu}$, the position of atom $i$ with respect to the center of mass $\mathbf{R}_{\mu}$ of bead $\mu, \mathbf{E}$ the identity matrix, and $\otimes$ the tensor product. The eigenvectors of the inertia tensor at time $t$ are denoted $\mathbf{u}_{\mu, 1}(t), \mathbf{u}_{\mu, 2}(t)$, and $\mathbf{u}_{\mu, 3}(t)$. The index $\mu$ is dropped for easy readability when possible.

To qualitatively compare the relaxation times of the first eigenvector $\mathbf{u}_{\mu, 1}(t)$ to those of known slow and fast variables, we compare their normalized autocorrelation functions (ACF). The normalized autocorrelation function of a vector $\mathbf{X}_{\mu}(t)$ depending on time $t$ and bead $\mu$ is defined as:

$$
\operatorname{ACF}(t)=\frac{\left\langle\mathbf{X}_{\mu}\left(t_{0}+t\right) \cdot \mathbf{X}_{\mu}\left(t_{0}\right)\right\rangle_{\mu, t_{0}}}{\left\langle\mathbf{X}_{\mu}\left(t_{0}\right) \cdot \mathbf{X}_{\mu}\left(t_{0}\right)\right\rangle_{\mu, t_{0}}},
$$

where $\langle\cdot\rangle_{\mu, t_{0}}$ denotes an average over all beads and all initial times $t_{0}$ and is the usual dot product. The ACFs are obtained in the NVE ensemble to prevent any artefact from a thermostat. The variables are evaluated every 10 fs to characterize rapidly relaxing variables. The normalized ACF of the first eigenvector $\mathbf{u}_{1}$ is compared to those of the velocity $\mathbf{V}$ and the force $\mathbf{F}$ of a bead in Fig. 2 (a) for different levels of coarse-graining, from 1 to 6 monomers per bead. The ACFs of the force $\mathbf{F}$ are starting at 1 and fluctuating around 0 after only a couple of points for all levels of coarse-graining. The results are nearly identical whether the average is done on a 500 ps time span or a 1 ns time span (not shown) indicating that the fluctuations seen are not due to noise but are significant. As points are separated by $10 \mathrm{fs}$, the order of magnitude of the force relaxation time should be around this value. This shows that the fluctuations of the force are indeed sensitive to fast atomic vibrations, which time scale is short and does not change as the level of coarse-graining increases. This result has been shown before on different systems 1 . On the contrary, the ACFs of the bead velocity $\mathbf{V}$ are still decaying after 10 ps. Moreover, it is decaying more and more slowly as the level of coarse-graining increases. For these two reasons, it can be safely considered as a slow variable. This has also been shown 
previously on different systems 4 . The ACFs of the eigenvector $\mathbf{u}_{1}$ have very similar features to those of the bead velocity $\mathbf{V}$ : it is still decaying after $100 \mathrm{ps}$, an even longer time than that of the velocity ACF, and it is decaying more and more slowly as the level of coarse-graining increases. Very similar trends are obtained for the two other eigenvectors (not shown). The bead orientation, defined as the eigenvectors of the inertia tensor, is consequently a slow variable and a complete coarse-graining description of the system cannot ignore this slow variable. The fact that the relaxation of the bead orientation is quite slow and slows down even more as the level of coarse-graining increases is not new in itself but its direct comparison to the relaxation of usual CG slow variables is. The fact that it is a slow variable in the same sense as the bead velocity is the first main result of the paper.

\subsubsection{Angular velocity.}

To elaborate a coarse-graining model which takes into account the bead orientation, we will need to follow its dynamics. We consequently define the angular velocity $\omega$ associated to the eigenvectors of the bead inertia tensor as the unique vector satisfying:

$$
\dot{\mathbf{u}}_{i}(t)=\omega_{\mu}(t) \times \mathbf{u}_{i}(t),
$$

where $\sqcup$ denotes the time derivative and $\times$ the usual cross product. For rigid bodies this equation can be applied to any atomic position with respect to the bead center of mass. In the general case of non-rigid bodies considered here, this can only be applied to the basis of eigenvectors of the inertia tensor. Mirroring what is done for the center of mass position and velocity in coarsegraining strategies, both the eigenvectors and the angular velocity will be considered as slow variables and their equations of motion need to be derived and averaged over fast atomic variables. The equation of motion for $\omega$ can be written as:

$$
\frac{d \mathbf{I}(t) \boldsymbol{\omega}(t)}{d t}=\mathbf{T}(t)
$$

For rigid bodies the vector $\mathbf{T}(t)$ is exactly the torque on bead $\mu$. For non-rigid bodies, which is the general case studied here, the vector $\mathbf{T}(t)$ is not the torque and is related to the force, position and velocity of each atom in a much more complicated way. However as the inertia tensor $\mathbf{I}$ and the angular velocity $\boldsymbol{\omega}(t)$ are clearly defined in terms of atomic variables, this relation does exist. By analogy to the case of rigid bodies, vector $\mathbf{T}$ will be named the effective torque. A useful and practical expression for the effective torque $\mathbf{T}$ is obtained by rearranging Eq. 4 as shown in the ESI†, Sec. S1:

$$
T_{i}^{\prime}=I_{i} \dot{\omega}_{i}^{\prime}+\dot{I}_{i} \omega_{i}^{\prime}-\omega_{i-1}^{\prime} \omega_{i+1}^{\prime}\left(I_{i+1}-I_{i-1}\right) \quad \text { for } i \in 1,2,3,
$$

where $T_{i}^{\prime}$ are the components of the effective torque $\mathbf{T}$ in the current basis of eigenvectors, $I_{i}$ is the $i^{\text {th }}$ moment of inertia and with the convention that $i+1$ and $i-1$ are the indices following and preceding $i$ in a circular permutation, respectively.

We have now defined three new types of variables per bead, the eigenvectors $\mathbf{u}_{1}, \mathbf{u}_{2}$ and $\mathbf{u}_{3}$, the angular velocity $\boldsymbol{\omega}$ and the effective torque $\mathbf{T}$. This is done in the idea that these new quantities have a similar role to play in the coarse-graining model as the
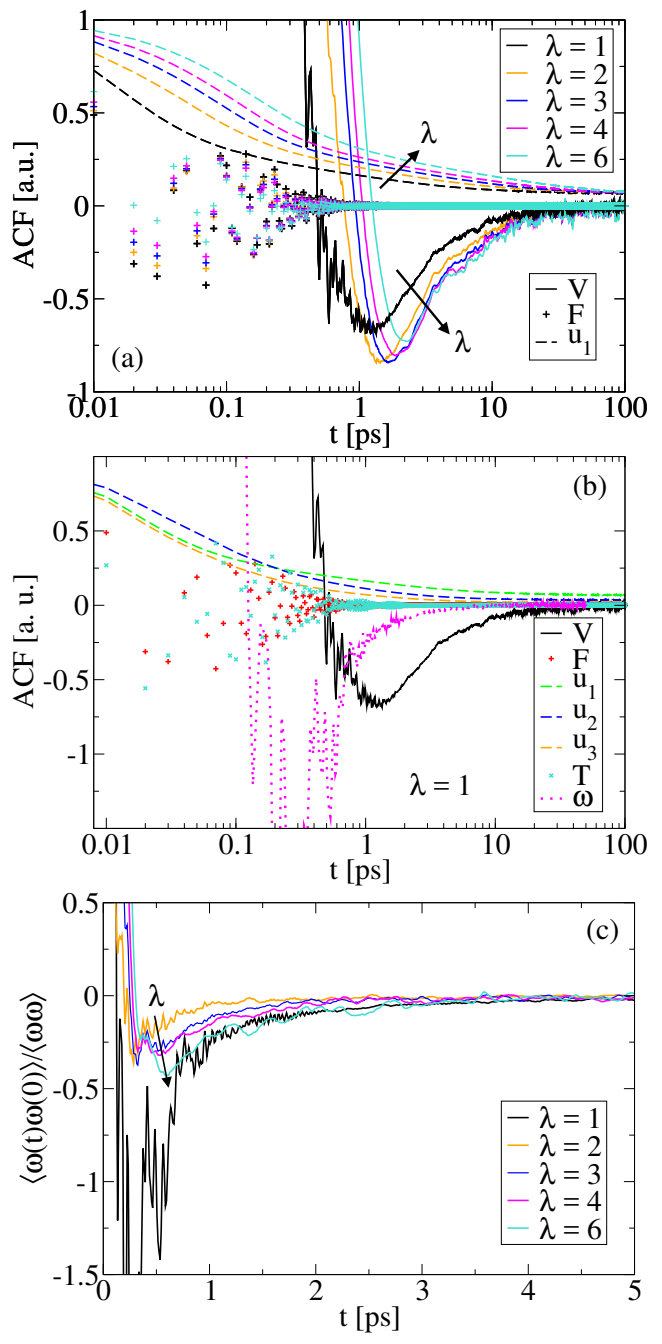

Fig. 2 (a) Time evolution of the normalized autocorrelation function (ACF), as defined in Eq. 2 of the velocity $\mathbf{V}$, force $\mathbf{F}$ and eigenvector $\mathbf{u}_{1}$ of a bead for different coarse-graining levels $\lambda$. The unit of the $y$-axis is arbitrary. The ACF of the velocity $\mathbf{V}$ is multiplied by 50 so that the evolution at long time is clearly visible on the same scale as the other ACFs. (b) Same as (a) for seven quantities the bead velocity $\mathbf{V}$, force $\mathbf{F}$, eigenvectors $\mathbf{u}_{1}, \mathbf{u}_{2}, \mathbf{u}_{3}$, angular velocity $\boldsymbol{\omega}$, and effective torque $\mathbf{T}$ for a level of coarse-graining $\lambda=1$. The ACF of the angular velocity $\omega$ is multiplied by 100 and the noise on this quantity is reduced using a moving average of size 10 . These modifications are done only to improve visibility. (c) Same as (a) and (b) for the angular velocity $\boldsymbol{\omega}$ at different levels of coarse-graining $\lambda$. 
center of mass position $\mathbf{R}$, the center of mass velocity $\mathbf{V}$ and the force $\mathbf{F}$, respectively. Before going into the details of the coarsegraining model, we need to check that the angular velocity $\omega$ can indeed be considered as a slow variable in the same sense as the velocity $\mathbf{V}$ and that the effective torque $\mathbf{T}$ is sensitive to fast atomic vibrations as the force F. Figure 2 (b) shows the normalized ACF of the angular velocity $\boldsymbol{\omega}$ and the effective torque $\mathbf{T}$ for a level of coarse-graining $\lambda$ of 1 monomer per bead. The ACFs of $\mathbf{V}, \mathbf{F}, \mathbf{u}_{1}, \mathbf{u}_{2}$ and $\mathbf{u}_{3}$, visible in Fig. 2 (a) are also reproduced in Fig. 2 (b) to facilitate the comparison. The ACF of the effective torque $\mathbf{T}$ has a very similar behavior as that of the force $\mathbf{F}$, it fluctuates around 0 after only a few tens of femtoseconds and can safely be considered as a fast variable like the force. The ACF of the angular velocity $\omega$ relaxes more quickly than that of the velocity $\mathbf{V}$, however it is still relaxing around $1 \mathrm{ps}$, when both the ACFs of the force $\mathbf{F}$ and the effective torque $\mathbf{T}$ are fluctuating around 0 . Thus, the angular velocity seems to be the fastest relaxing of all the slow variables but can still be considered as a slow variable. This is the second main result of this paper.

The variation of the relaxation of the angular velocity $\boldsymbol{\omega}$ with the level of coarse-graining is displayed in Fig. 2(c). Except for the case of $\lambda=1$ for which the angular velocity seems to be the most slowly relaxing, the angular velocity relaxes more and more slowly as the degree of coarse-graining increases, reinforcing the results that it is a slow variable in the same sense as the velocity of the center of mass.

\subsection{Full equations of motion in terms of atomic variables}

As explained in Sec. 3.1. we choose as slow variables the center of mass position $\mathbf{R}_{\mu}$ and velocity $\mathbf{V}_{\mu}$ of bead $\mu$ and also the eigenvectors $\mathbf{u}_{\mu, 1}, \mathbf{u}_{\mu, 2}$ and $\mathbf{u}_{\mu, 3}$ of the inertia tensor of bead $\mu$ and its angular velocity $\omega_{\mu}$. These variables are illustrated on a chain of cis-1,4-polybutadiene in Fig. 3 (a). Before deriving the coarse-grained equations of motion for these slow variables, it is necessary to make them explicit at the atomistic level.

The equations of motion for the center of mass position $\mathbf{R}_{\mu}$ and velocity $\mathbf{V}_{\mu}$ are straightforward and read

$$
\begin{aligned}
\dot{\mathbf{R}}_{\mu} & =\mathbf{V}_{\mu} \\
M_{\mu} \dot{\mathbf{V}}_{\mu} & =\mathbf{F}_{\mu},
\end{aligned}
$$

where $M_{\mu}=\sum_{i \in \mu} m_{i}$ is the mass of bead $\mu$ and $\mathbf{F}_{\mu}=\sum_{i \in \mu} \mathbf{f}_{i}$ is the sum of all forces $\mathbf{f}_{i}$

To get the equations of motion for the eigenvectors $\mathbf{u}_{\mu, 1}, \mathbf{u}_{\mu, 2}$ and $\mathbf{u}_{\mu, 3}$ and the angular velocity $\boldsymbol{\omega}_{\mu}$ a bit more work is necessary. The equation of motion of the eigenvectors can be expressed in a much more compact way using the corresponding quaternion $\mathrm{Q}_{\mu}$ as is done in the case of rigid bodies. More specifically, there is a single rotation which transforms the reference basis $\left(\mathbf{e}_{1}, \mathbf{e}_{2}, \mathbf{e}_{3}\right)$ of the laboratory frame into the current basis $\left(\mathbf{u}_{1}(t), \mathbf{u}_{2}(t), \mathbf{u}_{3}(t)\right)$. Its transpose, which corresponds to the opposite rotation, is denoted $\mathbf{C}(t)$ and satisfies:

$$
\boldsymbol{\omega}^{\prime}(t)=\mathbf{C}(t) \boldsymbol{\omega}(t)
$$

where $\boldsymbol{\omega}(t)$ is a vector, for example the angular velocity, which components are expressed in the reference basis $\left(\mathbf{e}_{1}, \mathbf{e}_{2}, \mathbf{e}_{3}\right)$ and $\boldsymbol{\omega}^{\prime}(t)$ is the same vector expressed in the current basis $\left(\mathbf{u}_{1}(t), \mathbf{u}_{2}(t), \mathbf{u}_{3}(t)\right)$. The rotation matrix $\mathbf{C}(t)$ is associated to the quaternion $\mathrm{Q}$ by

$\mathbf{C}(t)=\left[\begin{array}{ccc}Q_{0}^{2}+Q_{1}^{2}-Q_{2}^{2}-Q_{3}^{2} & 2\left(Q_{1} Q_{2}+Q_{0} Q_{3}\right) & 2\left(Q_{1} Q_{3}-Q_{0} Q_{2}\right) \\ 2\left(Q_{1} Q_{2}-Q_{0} Q_{3}\right) & Q_{0}^{2}-Q_{1}^{2}+Q_{2}^{2}-Q_{3}^{2} & 2\left(Q_{2} Q_{3}+Q_{0} Q_{1}\right) \\ 2\left(Q_{1} Q_{3}+Q_{0} Q_{2}\right) & 2\left(Q_{2} Q_{3}-Q_{0} Q_{1}\right) & Q_{0}^{2}-Q_{1}^{2}-Q_{2}^{2}+Q_{3}^{2}\end{array}\right]$

where the index $\mu$ is dropped for clarity and $Q_{i}$ for $i \in 0,1,2,3$ are the four components of the quaternion $\mathrm{Q}$. By construction quaternion $\mathrm{Q}$ has norm $\sum_{i=0}^{3} Q_{i}^{2}=1$.

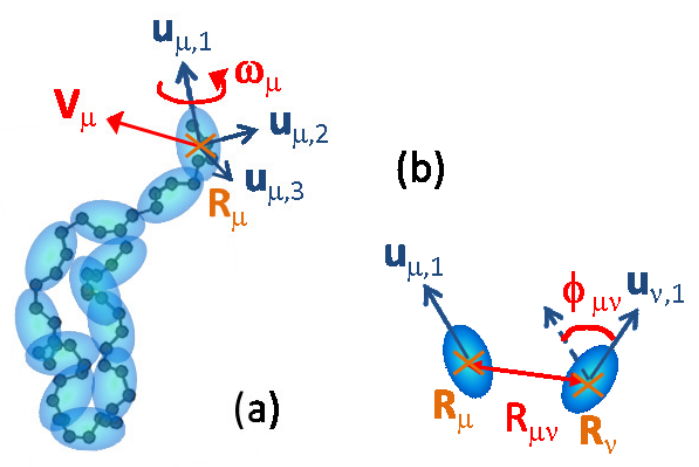

Fig. 3 (a) Illustration of the slow variables $\mathbf{R}_{\mu}, \mathbf{V}_{\mu}, \mathbf{u}_{\mu, 1}, \mathbf{u}_{\mu, 2}, \mathbf{u}_{\mu, 3}$, and $\boldsymbol{\omega}_{\mu}$ on a chain of cis-1,4-polybutadiene. (b) Illustration of the variables $R_{\mu \nu}$ and $\phi_{\mu \nu}$ relevant for the coarse-grained pair potential.

As shown in the ESI†, Sec. S2, and in a similar way to what is done for rigid bodies $\frac{40}{4}$, the equation of motion of the quaternion is:

$$
\dot{\mathrm{Q}}_{\mu}=\frac{1}{2} \mathrm{Aw}^{\prime}(t)
$$

where matrix $\mathbf{A}$ is

$$
\mathbf{A}=\left[\begin{array}{cccc}
Q_{0} & -Q_{1} & -Q_{2} & -Q_{3} \\
Q_{1} & Q_{0} & -Q_{3} & Q_{2} \\
Q_{2} & Q_{3} & Q_{0} & -Q_{1} \\
Q_{3} & -Q_{2} & Q_{1} & Q_{0}
\end{array}\right]
$$

and the quaternion $\mathbf{w}^{\prime}(t)$ is linked to the vector $\omega^{\prime}(t)$ by

$$
\mathrm{w}^{\prime}(t)=\left[\begin{array}{c}
0 \\
\omega_{1}^{\prime} \\
\omega_{2}^{\prime} \\
\omega_{3}^{\prime}
\end{array}\right]
$$

where $\omega_{i}^{\prime}$ for $i \in 1,2,3$ are the components of the angular velocity $\boldsymbol{\omega}(t)$ in the current basis $\left(\mathbf{u}_{1}(t), \mathbf{u}_{2}(t), \mathbf{u}_{3}(t)\right)$. Note here that a quaternion is denoted with a sans serif character like $Q$ while the usual convention of using bold characters for denoting vectors and matrices is used.

The equation of motion for $\boldsymbol{\omega}$ can be derived from Eq. 5 defining the effective torque:

$$
\dot{\omega}_{i}^{\prime}=\frac{T_{i}^{\prime}}{I_{i}}-\frac{\dot{I}_{i} \omega_{i}^{\prime}}{I_{i}}+\omega_{i+1}^{\prime} \omega_{i-1}^{\prime} \frac{\left(I_{i+1}-I_{i-1}\right)}{I_{i}} \text { for } i \in 1,2,3,
$$




\subsection{Coarse-grained equations of motion}

Eqs 6, 7, 10, and 13 are the full equations of motion for the relevant variables. They still depend on all atomic variables through the force, the effective torque and the inertia tensor of a bead. They need to be averaged under suitable approximations to integrate out effectively the atomic vibrations and keep only a dependence on the relevant variables. This work is thoroughly done for any relevant variable in Ref. ${ }^{4}$. Under the Markovian approximation, the relevant variables evolve much more slowly than the fast atomic vibrations. The generalized Langevin equation corresponding to Eq. (25) of Ref. 4 obtained under the Markovian approximation is for any set of slow variables $\alpha$ :

$$
\frac{d \boldsymbol{\alpha}(t)}{d t}=\nu(\boldsymbol{\alpha}(t))+\bar{M}(\boldsymbol{\alpha}(t)) \frac{\partial S}{\partial \boldsymbol{\alpha}}(\boldsymbol{\alpha}(t))+k_{B} \frac{\partial \bar{M}}{\partial \boldsymbol{\alpha}}(\boldsymbol{\alpha}(t))+\mathbf{R}(t),
$$

where $\bar{M}$ is a memory kernel, $S$ the entropy of the system, $\mathbf{R}$ a random white noise, and where

$$
\nu(\boldsymbol{\alpha})=\frac{1}{\Omega(\boldsymbol{\alpha})} \int \mathcal{L} \mathbf{A}(\mathbf{z}) \rho^{\mathrm{eq}}(\mathbf{z}) \delta(\mathbf{A}(\mathbf{z})-\boldsymbol{\alpha}) d \mathbf{z}
$$

and

$$
\mathcal{L} \mathbf{A}(\mathbf{z})=\frac{\partial \mathbf{a}}{\partial t}(t, \mathbf{z}), \quad \mathbf{a}(0, \mathbf{z})=\mathbf{A}(\mathbf{z})
$$

where $\mathbf{z}$ is the vector of all the microscopic variables and $\mathbf{a}(t, \mathbf{z})$ and $\mathbf{A}(\mathbf{z})$ are the slow variables expressed in terms of the microscopic variables z. The two notations do and do not highlight the time dependence, respectively. Vector $\boldsymbol{\alpha}$ is the considered set of values for the slow variable. $\mathcal{L}$ is the Liouville operator controlling the equation of motion of the slow variables and $\rho^{\mathrm{eq}}$ is the equilibrium probability density of the microscopic system. With words, Eq. 15 just means that $\nu(\boldsymbol{\alpha})$ is the average of the derivative $\partial \mathbf{a} / \partial t$ with respect to time of the slow variable $\mathbf{a}(t, \mathbf{z})$ over the fast degrees of freedom $\mathbf{z}$ for the fixed values $\boldsymbol{\alpha}$ of the slow variables $\mathbf{A}(\mathbf{z})$ at equilibrium. This average is denoted $\langle\partial \mathbf{a} / \partial t\rangle$ in the following. By construction, in Eq. 14 all functions depend only on the slow variables $\alpha$ and the effect of the fast variables is present only through the noise $\mathbf{R}$, which satisfies a fluctuation dissipation theorem:

$$
\langle\mathbf{R}(t) \mathbf{R}(s)\rangle=k_{B} \bar{M}(\alpha) \delta(t-s),
$$

where $k_{B}$ is the Boltzmann constant.

When there is a good time scale separation between the position $\mathbf{R}_{\mu}$ and velocity $\mathbf{V}_{\mu}$ of the bead center of mass and all other atomic variables, Eq. 14 reduces to ${ }^{4}$ :

$$
\begin{aligned}
\dot{\mathbf{R}}_{\mu} & =\mathbf{V}_{\mu} \\
M_{\mu} \dot{\mathbf{V}}_{\mu} & =\left\langle\mathbf{F}_{\mu}\right\rangle+\sum_{\nu} \gamma_{\mu \nu}\left(\mathbf{V}_{\mu}-\mathbf{V}_{\nu}\right)+k_{B} T \sum_{\nu} \frac{\partial \boldsymbol{\gamma}_{\mu \nu}}{\partial\left(M_{\mu} \mathbf{V}_{\mu}\right)}+\tilde{\mathbf{F}}_{\mu},
\end{aligned}
$$

where $\left\langle\mathbf{F}_{\mu}\right\rangle$ is the average of the force on bead $\mu$ for fixed values of all the slow variables $\mathbf{R}_{\nu}$ and $\mathbf{V}_{\nu}$ over the fast variables as defined in Eq. 15. $\gamma_{\mu \nu}$ is the friction tensor, and $\tilde{\mathbf{F}}_{\mu}$ is a white noise. This leads after some more approximations to the DPD equations of motion ${ }^{4}$.

In Sec. 3.1. we have shown that the bead orientation and angular velocity are also slow variables. In this work, we consequently assume that a good time scale separation is achieved when the slow variables are the positions $\mathbf{R}_{\mu}$, velocities $\mathbf{V}_{\mu}$, quaternions $\mathrm{Q}_{\mu}$ and angular velocity $\boldsymbol{\omega}_{\mu}$ of bead $\mu$. To simplify Eq. 14 further, we neglect the dissipative forces, in other words the memory kernel $\bar{M}$ is set to zero. As a consequence of Eq. 17 the noise is also zero. The corresponding equation will only be able to reproduce part of the structural properties of the atomic system but not its dynamical properties. Under those assumptions, Eq. 14 amounts to an average over the fast variables of the four equations of motion Eqs 6, 7, 10, and 13, This leads to:

$$
\begin{aligned}
\dot{\mathbf{R}}_{\mu} & =\mathbf{V}_{\mu}, \\
M_{\mu} \dot{\mathbf{V}}_{\mu} & =\langle\mathbf{F} \mu\rangle \\
\dot{\mathbf{Q}}_{\mu} & =\frac{1}{2} \mathbf{A} \mathbf{w}^{\prime}, \\
\dot{\omega}_{\mu, i}^{\prime} & =\left\langle\frac{T_{\mu, i}^{\prime}}{I_{i}}\right\rangle-\left\langle\frac{\dot{I}_{\mu, i} \omega_{\mu, i}^{\prime}}{I_{i}}\right\rangle+\omega_{\mu, i+1}^{\prime} \omega_{\mu, i-1}^{\prime}\left\langle\frac{\left(I_{i+1}-I_{i-1}\right)}{I_{i}}\right\rangle
\end{aligned}
$$

where the matrix $\mathbf{A}$ is defined in Eq. 11. To get Eq. 23 from the average of Eq. 13 , we used the fact that the average of the sum is the sum of the average and that the angular velocity $\omega_{\mu}^{\prime}$ expressed in the current basis is a slow variable, because $\omega_{\mu}$ is a slow variable by definition and the current basis is also a slow variable by definition.

We then make additional assumptions which drastically simplify Eq. 23. We assume that $\left\langle T_{\mu, i}^{\prime} / I_{i}\right\rangle=\left\langle T_{\mu, i}^{\prime}\right\rangle /\left\langle I_{i}\right\rangle$ and that the moments of inertia $\left\langle I_{i}\right\rangle$ do not depend on the slow variables and are always equal to their average value. To avoid any confusion between the average $\langle\cdot\rangle$ which usually depends on all the slow variables, we denote the average moments of inertia $\bar{I}_{i}$. We, then assume, that the second term on the right-hand side of Eq. $23\left\langle\dot{I}_{\mu, i} \omega_{\mu, i}^{\prime} / I_{i}\right\rangle$ can be neglected, which is consistent with the assumption that the moments of inertia are constant. In particular, they do not depend on time. Finally, for the third term on the right-hand side of Eq. 23, we assume that $\left\langle\left(I_{i+1}-I_{i-1}\right) /\left(I_{i}\right)\right\rangle=\left(\bar{I}_{i+1}-\bar{I}_{i-1}\right)\left(\bar{I}_{i}\right)$. The equation of motion of the angular velocity reads:

$$
\dot{\omega}_{\mu, i}^{\prime}=\frac{\left\langle T_{\mu, i}^{\prime}\right\rangle}{\bar{I}_{i}}+\omega_{\mu, i+1}^{\prime} \omega_{\mu, i-1}^{\prime} \frac{\left(\bar{I}_{i+1}-\bar{I}_{i-1}\right)}{\bar{I}_{i}} \quad \text { for } i \in 1,2,3 .
$$

To calculate the averages $\langle\cdot\rangle$ in Eqs. 21 and 24 , we make further assumptions. For the average force $\left\langle\mathbf{F}_{\mu}\right\rangle$, these assumptions are usual and similar to those done in Ref. 16 . Under the pair approximation, the force on bead $\mu$ is

$$
\left\langle\mathbf{F}_{\mu}\right\rangle=\sum_{\nu \neq \mu}\left\langle\mathbf{F}_{\mu \nu}\right\rangle
$$

where $\left\langle\mathbf{F}_{\mu \nu}\right\rangle$ is the average force due to bead $\nu$ on bead $\mu$. We 
further assume that the force $\left\langle\mathbf{F}_{\mu \nu}\right\rangle$ derives from a potential $W$ which depends only on the distance $R_{\mu \nu}$ between bead $\mu$ and $\nu$ and the angle $\phi_{\mu \nu}$ between the principal directions of bead $\mu$ and $\nu$. The angle $\phi_{\mu \nu}$ is chosen more specifically as the angle between the eigenvectors of the two beads associated to the lowest eigenvalue of the inertia tensor:

$$
\cos \phi_{\mu \nu}=\mathbf{u}_{\mu, 1} \cdot \mathbf{u}_{\nu, 1}
$$

We neglect the dependence on the positions, velocities and orientations of all other beads. The variables $R_{\mu \nu}$ and $\phi_{\mu \nu}$ relevant for the pair force are illustrated in Fig. 3 (b). The proposed potential $W$ is close to the popular Gay-Berne potential $\underline{41 / 42}$ but a little simpler: it depends only on one distance $R_{\mu \nu}$ and one angle $\phi_{\mu \nu}$ whereas the Gay-Berne potential depends on these two variables and also on two angles $\phi_{\mu}$ and $\phi_{\nu}$, defined as the angles between the vector linking the two centers of mass and the main direction of bead $\mu$ and $\nu$, respectively. This simplification allows the force to be in the same direction as in the isotropic case of a potential depending only on the distance, but with a different magnitude. Indeed, bearing in mind that $\partial \phi_{\mu \nu} / \partial \mathbf{R}_{\mu}=\mathbf{0}$, one can show that

$$
\left\langle\mathbf{F}_{\mu \nu}\right\rangle=-\frac{\partial W}{\partial R_{\mu \nu}}\left(R_{\mu \nu}, \phi_{\mu \nu}\right) \mathbf{e}_{\mu \nu},
$$

where $\mathbf{e}_{\mu \nu}=\left(\mathbf{R}_{\mu}-\mathbf{R}_{\nu}\right) /\left|\mathbf{R}_{\mu}-\mathbf{R}_{\nu}\right|$.

To extract the average effective torque $\left\langle\mathbf{T}_{\mu}^{\prime}\right\rangle$ of Eq. 24. we express it back into the reference basis:

$$
\left\langle\mathbf{T}_{\mu}\right\rangle=\sum_{i=1}^{3}\left\langle T_{\mu, i}^{\prime}\right\rangle \mathbf{u}_{\mu, i}
$$

The following assumptions are then made. The pair approximation for the effective torque leads to

$$
\left\langle\mathbf{T}_{\mu}\right\rangle=\sum_{\nu \neq \mu}\left\langle\mathbf{T}_{\mu \nu}\right\rangle
$$

Moreover, and similarly to the case of rigid bodies $43 / 44$, we assume that the torque $\left\langle\mathbf{T}_{\mu \nu}\right\rangle$ derives from the same potential as the force and depends only on the distance and angle between the bead principal directions through:

$$
\left\langle\mathbf{T}_{\mu \nu}\right\rangle=-\frac{\partial W}{\partial \cos \phi_{\mu \nu}} \mathbf{u}_{\mu, 1} \times \mathbf{u}_{\nu, 1} .
$$

\subsection{Computation of the coarse-grained anisotropic potential} $W$

To obtain the coarse-grained potential $W$ from atomistic simulations, we obtain its derivative with respect to the distance through Eq. 27 and its derivative with respect to the angle through Eq. 30 . For the derivative with respect to the distance, one has

$$
\frac{\partial W}{\partial R_{\mu \nu}}=-\left\langle\mathbf{F}_{\mu \nu}\right\rangle \cdot \mathbf{e}_{\mu \nu}
$$

and for the derivative with respect to the angle, one has

$$
\frac{\partial W}{\partial \cos \phi_{\mu \nu}}=-\frac{1}{\sin ^{2} \phi_{\mu \nu}}\left\langle\mathbf{T}_{\mu \nu}\right\rangle \cdot\left(\mathbf{u}_{\mu, 1} \times \mathbf{u}_{\nu, 1}\right),
$$

where the average $\langle\cdot\rangle$ is done for fixed values of the slow variables. The calculation of the average pair force $\left\langle\mathbf{F}_{\mu \nu}\right\rangle$ and the average pair effective torque $\left\langle\mathbf{T}_{\mu \nu}\right\rangle$ is detailed in the ESI $\dagger$, Sec. S3.

Once the two partial derivatives of the potential are obtained, the potential can be computed. This can be done by integration over the distance first and the angle then or the reverse. Integrating over the distance first and the angle then leads to

$W(R, \cos \phi)=\int_{R_{c}}^{R} d R^{\prime} \frac{\partial W}{\partial R}\left(R^{\prime}, \cos \phi\right)+\int_{0}^{\cos \phi} d C^{\prime} \frac{\partial W}{\partial \cos \phi}\left(R_{c}, C^{\prime}\right)$,

where the bound in distance is chosen to be the cutoff radius $R_{c}$ of the potential and the bound in $\cos \phi$ is chosen arbitrarily to be zero. Integrating over the angle first and the distance then and choosing the same bounds leads to

$$
W(R, \cos \phi)=\int_{0}^{\cos \phi} d C^{\prime} \frac{\partial W}{\partial \cos \phi}\left(R, C^{\prime}\right)+\int_{R_{c}}^{R} d R^{\prime} \frac{\partial W}{\partial R}\left(R^{\prime}, 0\right) .
$$

If the potential $W$ is an exact differential, Eqs. 33 and 34 will lead to the same result.
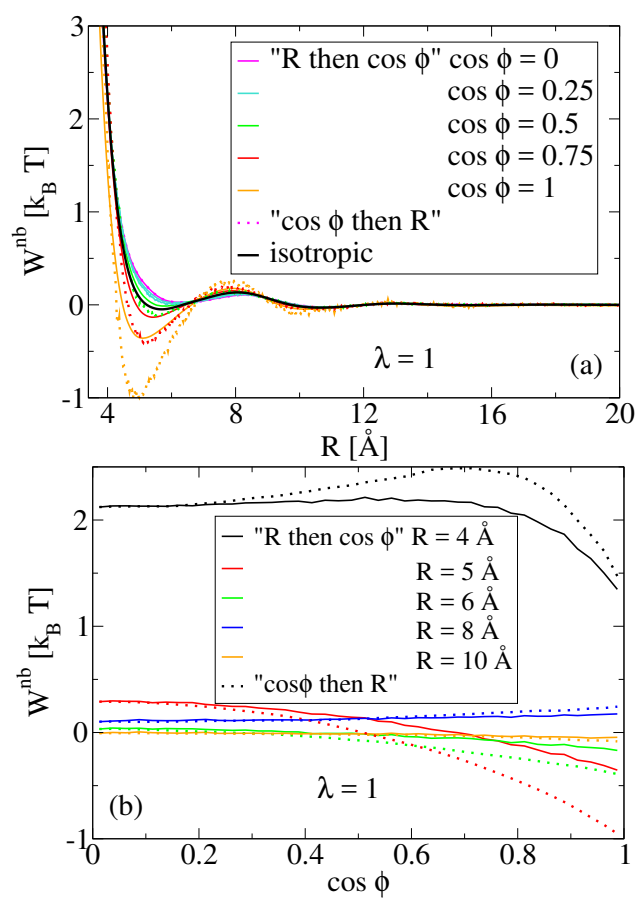

Fig. 4 (a) Variation of the non-bonded potential $W^{\text {nb }}$ with the distance $R$ for different values of $\cos \phi$ using the two integration paths given in Eq. 33 (solid lines) and Eq. 34 (dotted lines) for a level of coarsegraining $\lambda$ of 1 monomer per bead. The thick black lines correspond to the isotropic potential. (b) Same as (a) for the variation with $\cos \phi$ for different values of the distance $R$.

The results of the two integrations are compared for the representative case of the non bonded potentials $W^{\text {nb }}$ in Fig. 4 for a level of coarse-graining $\lambda$ of 1 monomer per bead. Figure 4 (a) shows the result of the two integrations versus the distance $R$ for different values of $\cos \phi$, and Fig. 4 (b) shows the same results 
versus $\cos \phi$ for different values of $R$. In both figures, one can see that there is a difference between the two integration paths. This difference increases for larger values of $\cos \phi$ because they lead to very close results at the bound value $\cos \phi=0$. In the same way, the difference between the two integration paths decreases as the distance gets closer to the bound value of $R=R_{c}$. Despite the differences seen between the two integration paths, the position of the peaks and the general trend of the potential versus $R$ and $\cos \phi$ are the same. We decided to keep the potential obtained by integration over the distance first and the angle then, because it appears the smoothest when plotted against $R$ and is still reasonably smooth when plotted against $\cos \phi$, whereas the other integration paths leads to more noisy results when the potential is plotted against $R$. Another important lesson learnt from Fig. 4 is that the effect of the relative orientation on the non-bonded potential is significant. In particular the relative first minimum around $5 \AA$ is still positive and purely repulsive for low values of $\cos \phi$ but becomes negative and attractive for $\cos \phi>0.68$. The energy difference between the lowest and highest values of $\cos \phi$ for this distance is of $0.65 k_{B} T$. The isotropic potential also displayed in Fig. 4 (a) and obtained without taking into account the relative orientations of the beads, is only slightly negative at this distance around $-0.05 k_{B} T$.

The integrated potential is then smoothed within empirically chosen bounds and extended to a continuous and differentiable function beyond the bounds. The empirically chosen bounds are useful to prevent smoothing regions scarcely visited in the course of a MD simulation where the noise is extremely high. This is true in particular for small distances for the non-bonded potential. The full smoothing procedure is described in the ESI $\dagger$, Sec. S4, and enables us to get a smoother function of both $R$ and $\cos \phi$ still very close to the raw data.

The full procedure to obtain the potential has just been outlined for the non bonded potential $W^{\mathrm{nb}}$. We apply the same procedure to intramolecular interactions. We consider two types of intramolecular interactions, those associated to two bonded beads and those associated to three consecutive bonded beads. The first type of interaction is usually a pair potential and depends on the bond length. It is straightforward to generalize it to a pair interaction depending on the distance $R$ between the two bonded beads and the angle $\phi$ between their two relative orientations. The results of the final smoothed and extended bonding potential $W^{\text {bond }}$ depending on both $R$ and $\cos \phi$ is shown in Fig. 5 (a) and (b) for a level of coarse-graining $\lambda$ of 1 monomer per bead. The effect of the relative orientation on the bonding potential is tremendous and non-trivial. From $\cos \phi=0$ to $\cos \phi=1$, the bonding potential plotted against $R$ evolves from a shape with a single central minimum around $R=3.5 \AA$, to a shape with two local minima around $R=3 \AA$ and $R=4.5 \AA$, and to a shape with a single minimum around $R=4.5 \AA$. At this distance the potential varies of more than $10 k_{B} T$ between the lowest and highest values of $\cos \phi$. The isotropic bonding potential, also displayed in Fig. 5 (a) has a large flat minimum between $R=3 \AA$ and $R=4.5 \AA$.

For intramolecular interactions between beads separated by two bonds, we computed the usual bending potential $W^{\text {bend }}$ from the angle distribution function $g(\cos \theta)$, where $\theta$ is the bending
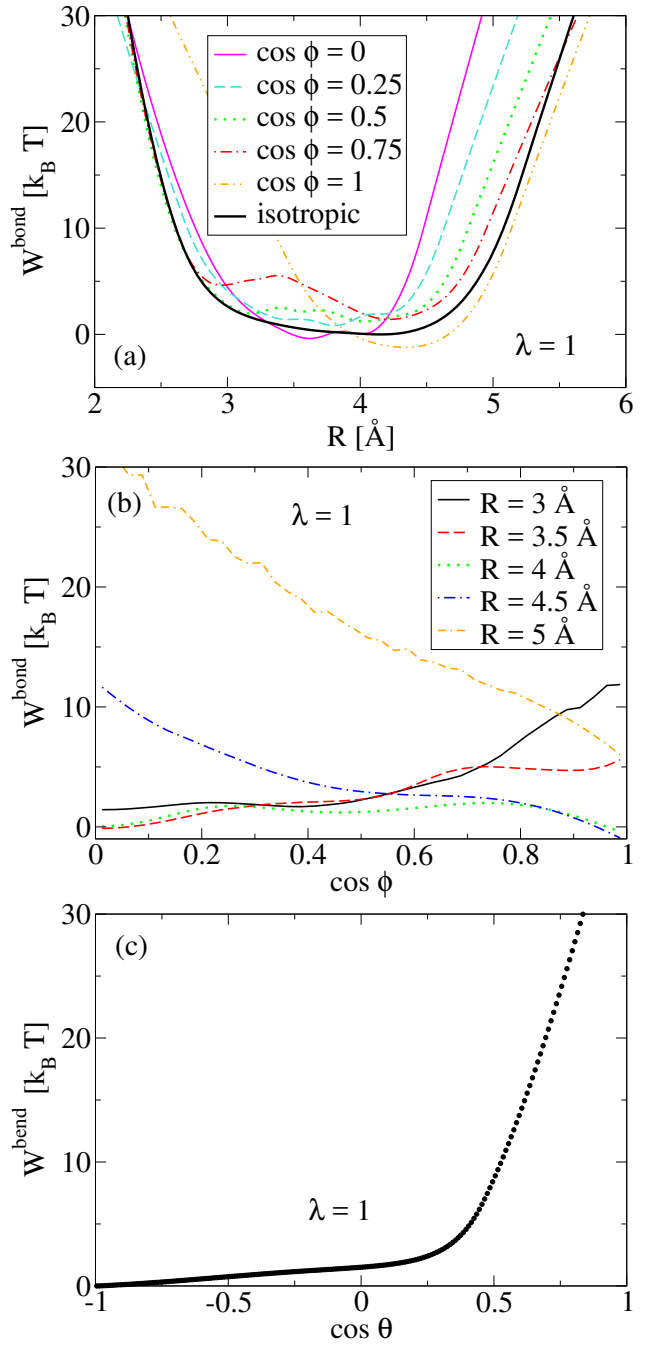

Fig. 5 (a) Variation of the bonding potential $W^{\text {bond }}$ with the distance $R$ for different values of $\cos \phi$. The thick black lines correspond to the isotropic potential. The results are shown after the smoothing and extension procedures have been applied. (b) Same as (a) for the variation with $\cos \phi$ at different values of $R$. (c) Variation of the classical bending potential $W^{\text {bend }}$ with $\cos \theta$, where $\theta$ is the bending angle, for a level of coarse-graining $\lambda$ of 1 monomer per bead.

angle between the three consecutive beads, as done in many works 1645146 . The effect of the anisotropy on the bending potential is not considered in this work. The final results after the smoothing procedure is shown in Fig 5 (c) for a level of coarsegraining $\lambda$ of 1 monomer per bead. As can be expected, this potential favors wide bending angles, as wide as $180^{\circ}$ and disfavors acute bending angles.

Once all non bonded and intramolecular potentials have been derived, the full coarse-grained potential $W^{\mathrm{CG}}$ can be expressed:

$$
\begin{aligned}
W^{\mathrm{CG}} & =\sum_{\mu \nu, \mathrm{nb}} W^{\mathrm{nb}}\left(R_{\mu \nu}, \cos \phi_{\mu \nu}\right)+\sum_{\mu \nu, \text { bond }} W^{\text {bond }}\left(R_{\mu \nu}, \cos \phi_{\mu \nu}\right) \\
& +\sum_{\mu \nu, \text {, bend }} W^{\text {bend }}\left(\cos \theta_{\mu \nu o}\right),
\end{aligned}
$$


where $\sum_{\mu \nu, \mathrm{nb}}$ and $\sum_{\mu \nu \text {,bond }}$ denote the sums on pairs of beads which are non bonded and directly bonded, respectively and where $\sum_{\mu \nu o, \text { bend }}$ is the sum over triplets of particles in a bending angle and $\theta_{\mu \nu o}$ the corresponding bending angle.

The equations of motion Eqs. 20 22 and 24 and the CG potential defined by Eqs 3133 form the anisotropic CG model. Note that a complementary approach to the one presented in this work has been very recently developed by Martzel et al ${ }^{47}$, also in the idea to take into account the bead anisotropy. Similarly to our choice of the inertia tensor, they use the tensor of gyration to describe the bead anisotropy. In contrast to our approximations, they keep the eigenvalues of the deviatoric part of the tensor of gyration and not its eigenvectors as the relevant variables. They choose an ad hoc expression for the anisotropic CG potential energy, fitted to the atomistic probability of each eigenvalue. Using this expression, they derive the conservative, dissipative and random contributions of the CG equations of motion for the center of mass position and the tensor of gyration. Compared to the

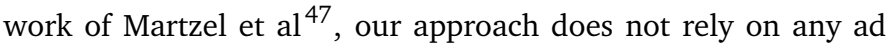
hoc expression for the CG potential, which allows us to test thoroughly the validity of all the approximations made to obtain the CG model. This is the purpose of Sec. 5. Nevertheless, the expressions proposed for the dissipative and random contributions to the equations of motion in Ref. $\frac{47}{4}$ are insightful and including them in our approach is an exciting perspective for future work.

\section{Comparison of structural properties in MD and CG simulations}

As the dissipative and random forces have been neglected in the derivation of the coarse-grained equations of motion $20,21,22$ 24 the coarse-grained simulations can only be expected to reproduce the structural properties of the underlying atomistic simulations. Two kinds of structural properties are compared, structural properties defined at the atomic level such as the usual radial distribution functions and the radial and angular distribution functions, and structural properties of the polymer chains such as their end-to-end distances and radii of gyration. For all these structural properties the comparison is carried out at three levels of coarse-graining from 1 to 3 monomers per bead.

\subsection{Radial and angular distribution functions}

To check that the anisotropic CG procedure is able to reproduce the locally anisotropic structure of the polybutadiene melt, we compare the non-bonding radial and angular distribution function $g^{\mathrm{nb}}(R, \cos \phi)$ obtained in MD and in anisotropic CG simulations. The distribution function $g^{\mathrm{nb}}(R, \cos \phi)$ is defined as:

$$
g^{\mathrm{nb}}(R, \cos \phi)=P(R, \cos \phi) / N(R, \cos \phi),
$$

where $P(R, \cos \phi)$ is the probability, calculated in a simulation, that two particles are at a distance $R$ and have a relative orientation of $\cos \phi$ and $N(R, \cos \phi)$ is the same probability in a homogeneous mixture. In a homogeneous mixture, the distribution in $\cos \phi$ is uniform, so that for all $\cos \phi$ one has $N(R, \cos \phi) \sim$ $V_{\text {shell }}(R)$, where $V_{\text {shell }}(R)$ is the volume of the shell centered on a particle and bounded by the two spheres of radii $R$ and $R+d R$, respectively, for the chosen binning $d R$. The term "non-bonding" refers to pairs of particles which are considered non-bonded by the coarse-grained potential, which are, in our case, particles in different molecules and particles in the same molecule beyond and including 1,4 interactions. The non-bonding distribution function $g^{\mathrm{nb}}(R, \cos \phi)$ obtained in MD and anisotropic CG simulations are displayed for the three levels of coarse-graining $\lambda$ in Fig. 6 The differences between each CG result and the corresponding MD result are also displayed at the bottom of each figure. The anisotropic CG simulations are able to reproduce quite well the huge differences observable as $\cos \phi$ increases from 0 to 1 on the radial distribution functions $g^{\mathrm{nb}}(R, \cos \phi)$ obtained in MD simulations, without any fitting parameter. This is the third main result of the paper. Even if the agreement between MD and anisotropic CG simulations is generally good when it comes to the non-bonding distribution function $g^{\mathrm{nb}}(R, \cos \phi)$, there are some differences. For all levels of coarse-graining, the difference is always the highest around the first peak for $\cos \phi=1$, when particles are parallel to each other. This configuration is always overrepresented in the anisotropic coarse-grained simulations. This difference between MD and CG results tend to increase as the level of coarse-graining increases. In contrast, the too large overlap seen in CG simulations between beads at short distances, especially for $\lambda=3$, is mainly due to small values of $\cos \phi \leq 0.5$. For $\cos \phi=1$, the difference with the MD results at short distances $R<4 \AA$ is zero.

\subsection{Radial distribution functions}

Now that we have checked that the anisotropic CG simulations are indeed able to reproduce the structural properties which they are designed to model, we can compare their predictions with those of the more usual isotropic CG simulations. The nonbonding radial distribution function is defined as usual as:

$$
g^{\mathrm{nb}}(R)=P(R) / N(R),
$$

where $P(R)$ is the probability, calculated in a MD or CG simulation, that two particles are at a distance $R$, and $N(R)$ is a normalization factor equal to the probability to find two particles at that distance in a homogeneous mixture. One has for all $\phi$, $N(R, \cos \phi)=N(R)$ and consequently

$$
g^{\mathrm{nb}}(R)=\frac{1}{N_{\phi}} \sum_{\phi} g^{\mathrm{nb}}(R, \cos \phi),
$$

where $N_{\phi}$ is the number of bins in $\cos \phi$. The non-bonding radial distribution functions $g^{\mathrm{nb}}(R)$ obtained in MD and in isotropic and anisotropic CG simulations are displayed in Fig. 7 for the three different levels of coarse-graining $\lambda$. The unfortunate result shown in Fig. 7 is that the non-bonding radial distribution functions obtained in isotropic and anisotropic CG simulations are quasi-identical. On can notice a small difference between the two results at very small distances where the radial distribution function varies a lot and the statistics decreases. One can also notice a very slight difference, which just exceeds the noise level, around the first peak, for all levels of coarse-graining. This is illustrated 

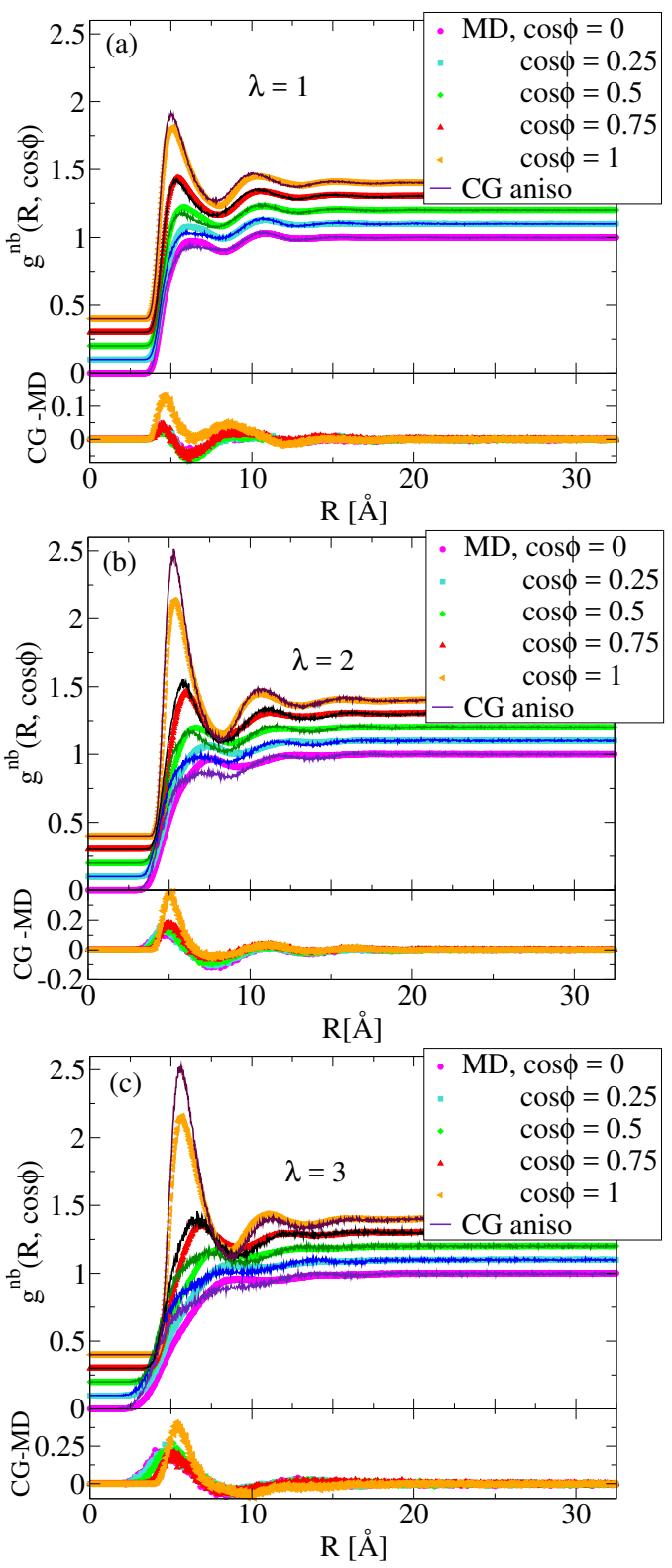

Fig. 6 (a) Non-bonding radial and angular distribution functions $g^{\mathrm{nb}}(R, \cos \phi)$ obtained in MD and anisotropic CG simulations for a coarsegraining level of $\lambda=1$ as a function of the distance $R$ between two beads and for different values of $\cos \phi$. The MD results are displayed using symbols, the corresponding CG result are displayed using a solid line of a darker shade of the same color. To improve readability the results have been gradually shifted up from $\cos \phi=0$ (the lowest curves) to $\cos \phi=1$ (the highest curves). The bottom figure displays the difference between each anisotropic CG result and the corresponding MD result for a given value of $\cos \phi$. The color code is the same as that in the main figure. (b) and (c) same as (a) for a level of coarse-graining $\lambda$ of 2 and 3 , respectively. in the zoomed plots in the insets of Fig. 7 In any case, the difference between the two CG radial distribution functions is much smaller than that between the CG and the MD distribution functions. This leads us to conclude that the discrepancy between the MD and CG $g^{\mathrm{nb}}(R)$ is not due to the anisotropy, at least not in the way it was taken into account in this work.

To investigate further the reason why the anisotropic CG simulations lead to the same radial distribution functions as the isotropic ones whereas the effect of the orientation on the potential is important, we study the conditional probability $P_{R}(\cos \phi)$ for two particles to have a relative orientation $\cos \phi$ given that they are at a distance of $R$. The conditional probability satisfies:

$$
\begin{aligned}
P_{R}(\cos \phi) & =\frac{P(R, \cos \phi)}{P(R)}, \\
& =\frac{g^{\mathrm{nb}}(R, \cos \phi)}{g^{\mathrm{nb}}(R)} .
\end{aligned}
$$

Equation 40 is obtained using the fact that the normalization constants are the same: $N(R, \cos \phi)=N(R)$, for all $\phi$. Equation 40 yields a practical way to compute the conditional probabilities in MD and anisotropic CG simulations. They are displayed as a function of the distance $R$ for different values of $\cos \phi$ for the three levels of coarse-graining $\lambda$ in Fig. 8 The differences between the MD and anisotropic CG results are also displayed at the bottom of each figure. It is clear from this figure that the differences between the MD and anisotropic CG results are smaller and less systematic for the conditional probability $P_{R}(\cos \phi)$ than for the distribution function $g^{\mathrm{nb}}(R, \cos \phi)$. The differences between MD and anisotropic CG results for the conditional probability $P_{R}(\cos \phi)$ are on the order of the noise level on this quantity and no clear bias as a function of $R$ or $\cos \phi$ is visible. There are a few exceptions however: for $\lambda=1$ and $\cos \phi=1$ the difference between the two results is on the order of magnitude of the difference between the distribution functions $g^{\mathrm{nb}}(R, \cos \phi)$; for $\lambda=2$ and $\cos \phi \leq 0.25$, and for $\lambda=3$ and $\cos \phi=1$ also. These exceptions are very contained and globally the anisotropic CG simulations are able to reproduce extremely well the conditional probability $P_{R}(\cos \phi)$.

The fact that anisotropic CG simulations are so good at reproducing the conditional probability $P_{R}(\cos \phi)$ explains why the isotropic and anisotropic coarse-grained $g^{\mathrm{nb}}(R)$ are nearly identical in the framework considered in this work to take into account the anisotropy. A short proof follows. In anisotropic CG simulations, the average non-bonding force $\left\langle F^{\text {aniso }}\right\rangle$ is calculated as described in Sec. 3.4 .

$$
\left\langle F^{\mathrm{aniso}}\right\rangle(R, \cos \phi)=\frac{\sum_{(\mu, \nu)(R, \cos \phi)} F_{\mu \nu}^{\mathrm{MD}}}{N_{\text {pairs }}(R, \cos \phi)},
$$

where $F_{\mu \nu}^{\mathrm{MD}}=\mathbf{F}_{\mu \nu} \cdot \mathbf{e}_{\mu \nu}$ is the norm of the force obtained in the MD simulation between beads $\mu$ and $\nu$ in a given configuration, $\sum_{(\mu, \nu)(R, \cos \phi)}$ is a sum over all pairs of beads, observed in the course of an MD simulation, located at a distance $R$ of each other and with the relative orientation $\cos \phi$, and $N_{\text {pairs }}(R, \cos \phi)$ is the number of such pairs encountered during the whole MD simula- 

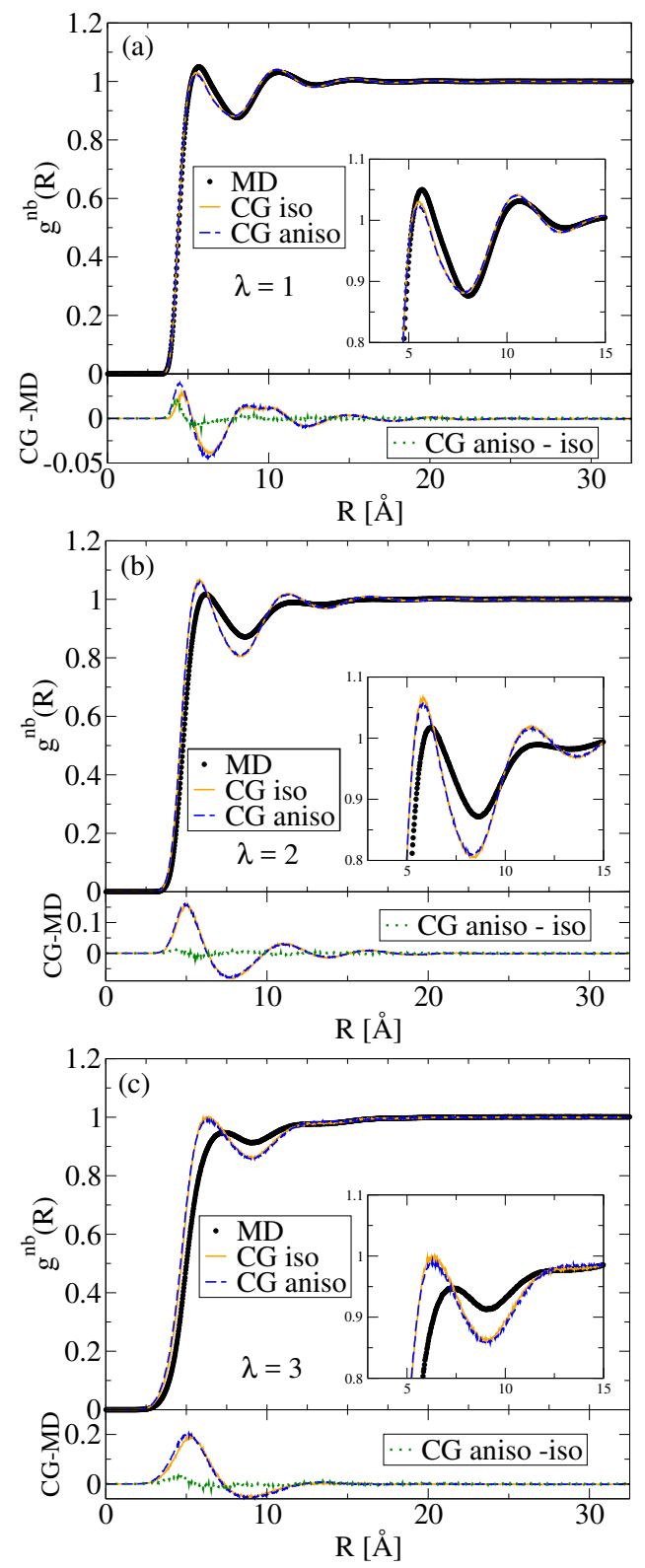

Fig. 7 (a) Non-bonding radial distribution functions $g^{\mathrm{nb}}(R)$ obtained in MD (black circles), isotropic (orange solid line) and anisotropic (blue dashed line) CG simulations for a coarse-graining level of $\lambda=1$ as a function of the distance $R$ between two beads. The inset is a zoom around the first two peaks. The bottom figure displays the difference between the isotropic CG result and the MD result (orange solid line), the anisotropic CG result and the MD result (blue dashed line) and the two CG results (green dotted line). (b) and (c) same as (a) for levels of coarse-graining $\lambda$ of 2 and 3 , respectively. tion. In the same way, the isotropic average non-bonding force $\left\langle F^{\mathrm{iso}}\right\rangle$ is:

$$
\left\langle F^{\text {iso }}\right\rangle(R)=\frac{\sum_{(\mu, \nu)(R)} F_{\mu \nu}^{\mathrm{MD}}}{N_{\text {pairs }}(R)},
$$

where $\sum_{(\mu, \nu)(R)}$ is a sum over all pairs of beads located at a distance $R$ of each other, and $N_{\text {pairs }}(R)$ is the number of such pairs encountered during the whole MD simulation. Obviously, as the same MD simulation is used to derive the isotropic and anisotropic CG forces, the same forces $F_{\mu \nu}^{\mathrm{MD}}$ are used in both cases. Moreover, by definition of the two sums, we have $\sum_{(\mu, \nu)(R)}=\sum_{\cos \phi} \sum_{(\mu, \nu)(R, \cos \phi)}$, where $\sum_{\cos \phi}$ is a sum over all possible bins of $\cos \phi$. Thus, we can express the isotropic CG force defined in Eq. 42 in terms of the anisotropic one:

$$
\begin{aligned}
\left\langle F^{\mathrm{iso}}\right\rangle(R) & =\frac{1}{N_{\text {pairs }}(R)} \sum_{\cos \phi} \sum_{(\mu, \nu)(R, \cos \phi)} F_{\mu \nu}^{\mathrm{MD}}, \\
& =\frac{1}{N_{\text {pairs }}(R)} \sum_{\cos \phi} \frac{\sum_{(\mu, \nu)(R, \cos \phi)} F_{\mu \nu}^{\mathrm{MD}}}{N_{\text {pairs }}(R, \cos \phi)} N_{\text {pairs }}(R, \cos \phi), \\
& =\sum_{\cos \phi} \frac{\sum_{(\mu, \nu)(R, \cos \phi)} F_{\mu \nu}^{\mathrm{MD}}}{N_{\text {pairs }}(R, \cos \phi)} \frac{N_{\text {pairs }}(R, \cos \phi)}{N_{\text {pairs }}(R)} \\
& =\sum_{\cos \phi}\left\langle F^{\text {aniso }}\right\rangle(R, \cos \phi) P_{R}^{\mathrm{MD}}(\cos \phi),
\end{aligned}
$$

by definition of the anisotropic CG force Eq. 41 and where $P_{R}^{\mathrm{MD}}(\cos \phi)$ is the conditional probability obtained in the MD simulations for two beads to have a relative orientation of $\cos \phi$ given that they are at a distance $R$. Now, if we write down the equations of motion for the velocity of a bead in the isotropic CG simulations, we get:

$$
\begin{aligned}
M \dot{\mathbf{V}}_{\mu} & =\sum_{\nu}\left\langle F^{\text {iso }}\right\rangle\left(R_{\mu \nu}\right) \mathbf{e}_{\mu \nu} \\
& =\sum_{\nu}\left(\sum_{\cos \phi}\left\langle F^{\text {aniso }}\right\rangle\left(R_{\mu \nu}, \cos \phi\right) P_{R_{\mu \nu}^{\mathrm{MD}}}(\cos \phi)\right) \mathbf{e}_{\mu \nu}
\end{aligned}
$$

according to Eq. 46, and in the anisotropic simulations, we have:

$$
M \dot{\mathbf{V}}_{\mu}=\sum_{\nu}\left\langle F^{\text {aniso }}\right\rangle\left(R_{\mu \nu}, \cos \phi_{\mu \nu}\right) \mathbf{e}_{\mu \nu}
$$

If in the anisotropic CG simulations the probability for two beads to have a relative orientation $\cos \phi$ given that they are at a distance $R$ of each other is the same as in the MD simulations, then on average all beads describe the same trajectories in the $(\mathbf{R}, \mathbf{V})$ space in the isotropic and anisotropic CG simulations. As a consequence the radial distribution functions in both cases are the same. This is what happens in this work.

A slightly different situation would occur if the anisotropy was taken into account in a different way. As done in the GayBerne potential $\frac{4142}{4}$, we could assume that the anisotropic potential also depends on the angles $\phi_{\mu}$ and $\phi_{\nu}$ defined as $\cos \phi_{\mu}=$ 
$\mathbf{u}_{\mu, 1} \cdot \mathbf{e}_{\mu \nu}$ and $\cos \phi_{\nu}=-\mathbf{u}_{\nu, 1} \cdot \mathbf{e}_{\mu \nu}$. This has been done in different coarse-grained anisotropic potentials designed over the years $23,25[48 / 49$. This assumption, changes the orientation of the force, which now have contributions along $\partial \cos \phi_{\mu} / \partial \mathbf{R}_{\mu}$ and $\partial \cos \phi_{\nu} / \partial \mathbf{R}_{\mu}$, and not only along $\mathbf{e}_{\mu \nu}$. We kindly refer the reader to Sec. 5.5 where the main directions of the force and torque are further tested and discussed.
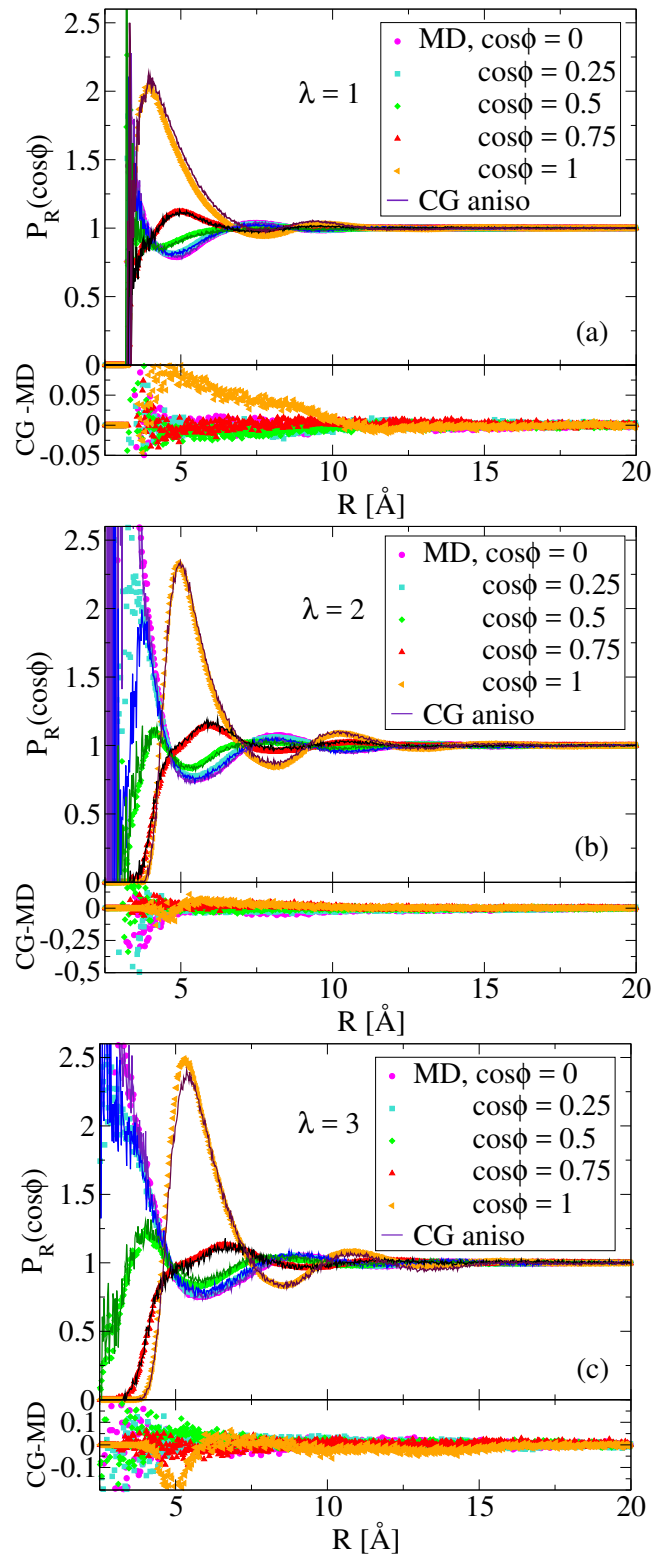

Fig. 8 Same legend as Fig. 6 for the conditional probability $P_{R}(\cos \phi)$.

\subsection{Chain dimensions}

To complement the comparison between the structural properties obtained in MD, isotropic and anisotropic CG simulations, we also looked at the end-to-end distance and radius of gyration of the chains. The end-to-end distance and radius of gyration are calculated for each level of coarse-graining using the position of the bead center of mass both in CG simulations, where it is the

\begin{tabular}{lcccc}
\hline$\lambda$ & {$[\AA]$} & MD & CG iso & CG aniso \\
\hline 1 & $\left\langle R_{\mathrm{ee}}\right\rangle$ & $20.00 \pm 0.05$ & $21.33 \pm 0.04$ & $21.3 \pm 0.1$ \\
2 & & $18.86 \pm 0.05$ & $18.21 \pm 0.02$ & $18.27 \pm 0.04$ \\
3 & & $17.57 \pm 0.04$ & $16.63 \pm 0.02$ & $16.46 \pm 0.03$ \\
\hline 1 & $\left\langle R_{\mathrm{g}}\right\rangle$ & & & \\
2 & & $8.29 \pm 0.01$ & $8.52 \pm 0.01$ & $8.51 \pm 0.03$ \\
3 & & $8.01 \pm 0.01$ & $7.59 \pm 0.01$ & $7.60 \pm 0.01$ \\
\hline
\end{tabular}

Table 2 Values of the average end-to-end distance $\left\langle R_{\mathrm{ee}}\right\rangle$ and radius of gyration $\left\langle R_{\mathrm{g}}\right\rangle$ obtained in $\mathrm{MD}$, isotropic and anisotropic CG simulations for different levels of coarse-graining $\lambda$. Each simulation lasts $10 \mathrm{~ns}$ and is sampled every $100 \mathrm{fs}$ in MD and every $1 \mathrm{ps}$ in CG simulations. The indicated error is the plateau value of the standard error on the mean evaluated for different numbers of blocks from 5 to 500

only possible thing to do, and in MD simulations, as defined in Ref. ${ }^{16}$ to obtain directly comparable results. These results are given in Table 2 Similarly to the results on the radial distribution functions, one can notice two things. The first is that the isotropic and anisotropic CG simulations lead very close structural properties of the chains. The results are barely distinguishable within error bars. This is likely due the same reason as that explaining the similarity of the radial distributions functions obtained in both CG simulations : the average trajectories in the $(\mathbf{R}, \mathbf{V})$ space of the beads are similar in isotropic and anisotropic simulations. The second thing to notice is that the difference between the CG and MD results changes sign as the level of coarse-graining $\lambda$ increases: the chain dimensions are larger in CG simulations than in MD simulations for a level of coarse-graining of 1 but are smaller than in MD for higher levels of coarse-graining. The smallest difference is obtained for $\lambda=2$ as was already noticed $i n$. The results on the chain dimensions confirms the conclusion drawn from the radial distribution functions, the anisotropy of the bead, although important, is not the reason for the discrepancy seen between MD and CG results, at least not in the way it was taken into account in this work.

\section{Verification of the approximations and lim- its of the model}

To justify and apprehend the limits and possible improvements of the model developed in Sec. 3we carefully check and discuss the approximations made to derive it. We do so in the order in which the approximations are made from the first and most important one to the last and most easily adaptable one.

\subsection{Markovian approximation}

The Markovian approximation is primordial as the whole framework of the model relies on it. It assumes that there is a clear time scale separation between the slow and fast variables. In the case of the anisotropic coarse-grained simulations, the slow variables are the position $\mathbf{R}_{\mu}$ and velocity $\mathbf{V}_{\mu}$ of the bead center of mass and its quaternion $Q_{\mu}$ and angular velocity $\boldsymbol{\omega}_{\mu}$. The fact that these variables are indeed slow was checked prior to the development of the CG model in Sec. 3.1 


\subsection{Derivative of the principal moments of inertia with re- spect to time}

The second most important assumption, is that Eq. 24 holds. This enables us to use any algorithm developed for rigid bodies to integrate the CG equations of motion. To show that Eq. 24 holds, we should show that the derivative with respect to time of the moments of inertia can be neglected before the derivative of the angular velocity. To improve readability, we define the two vectors $\mathbf{W}$ and $\epsilon$ as

$$
\begin{aligned}
W_{i} & =\dot{\omega}_{\mu, i}^{\prime} \\
\epsilon_{i} & =\frac{\dot{I}_{\mu, i} \omega_{\mu, i}^{\prime}}{I_{i}}, \quad \text { for } i \in 1,2,3 .
\end{aligned}
$$

for any bead $\mu$. We want to show that $\epsilon$ can be neglected before $\mathbf{W}$. To do so, we calculate the two averaged quantities: $(\mathbf{W}-\boldsymbol{\epsilon})^{2} / \mathbf{W}^{2}$ and $\boldsymbol{\epsilon}^{2} / \mathbf{W}^{2}$, during an NVT trajectory of $500 \mathrm{ps.}$ The results are shown in Table 3 for all levels of coarse-graining. Clearly $(\mathbf{W}-\boldsymbol{\epsilon})^{2} / \mathbf{W}^{2}$ tends to 1 and $\boldsymbol{\epsilon}^{2} / \mathbf{W}^{2}$ tends to 0 at all levels of coarse-graining, showing that neglecting the derivative with respect to time of the moments of inertia is meaningful. The results are even improved for larger levels of coarse-graining. This the fourth main result of this work.

\begin{tabular}{lcc}
\hline$\lambda$ & $(\mathbf{W}-\boldsymbol{\epsilon})^{2} / \mathbf{W}^{2}$ & $\boldsymbol{\epsilon}^{2} / \mathbf{W}^{2}$ \\
\hline 1 & 0.991 & $9.0 \times 10^{-3}$ \\
2 & 0.9998 & $4.5 \times 10^{-5}$ \\
3 & 0.9997 & $2.9 \times 10^{-5}$ \\
\hline
\end{tabular}

Table 3 Values of the quantities $(\mathbf{W}-\boldsymbol{\epsilon})^{2} / \mathbf{W}^{2}$ and $\epsilon^{2} / \mathbf{W}^{2}$ defined in Eqs. 50 and 51 for different levels of coarse-graining $\lambda$

\subsection{Bead shape}

Since we make the assumption that the coarse-grained potential depends mainly on the distance between two beads and the angle defined as $\cos \phi_{\mu \nu}=\mathbf{u}_{\mu, 1} \cdot \mathbf{u}_{\nu, 1}$, where $\mathbf{u}_{\mu, 1}$ and $\mathbf{u}_{\nu, 1}$ are the eigenvectors of the two beads associated to the lowest eigenvalue $I_{1}$, we need to check that selecting this specific orientation is meaningful. The three principal moments of inertia are sorted so that: $I_{3}>I_{2}>I_{1}$. If $\mathbf{D}_{I}$ corresponds to the diagonal matrix whose non-zero elements are the moments of inertia, the equation

$$
\mathbf{r}^{T} \mathbf{D}_{I} \mathbf{r}=1
$$

defines the ellipsoid enveloping the bead of principal radii $1 / \sqrt{I_{1}}$, $1 / \sqrt{I_{2}}$ and $1 / \sqrt{I_{3}}$. Thus, if one eigenvalue is significantly larger than the two others, one principal radius is significantly smaller than the others, implying that the particle is rather flat. In contrast, if one eigenvalue is significantly smaller than the two others, the bead is rather elongated. We have calculated the eigenvalues of the inertia tensor from an NVT trajectory of $500 \mathrm{ps}$ and averaged over all beads and time. Results show that $I_{1}<I_{2} \sim I_{3}$ as can be seen in Table 4 for all levels of coarse-graining. In particular, the aspect ratio $I_{3} / I_{1}$ is between 5 and 7 depending on the level of coarse-graining. It shows that the coarse-grained particles are elongated in the direction of the eigenvector associated to the smallest eigenvalue $I_{1}$, this is why choosing this eigenvector as representative of the bead orientation appears meaningful.

\begin{tabular}{lcccc}
\hline$\lambda$ & {$\left[\mathrm{kg} / \mathrm{mol} . \AA^{2}\right]$} & $I_{1}$ & $I_{2}$ & $I_{3}$ \\
\hline 1 & 0.020 & 0.081 & 0.10 \\
2 & 0.092 & 0.57 & 0.62 \\
3 & 0.27 & 1.6 & 1.8 \\
\hline
\end{tabular}

Table 4 Values of the three moments of inertia for different levels of coarse-graining $\lambda$ obtained in an NVT simulation

\subsection{Pair approximation}

Strictly speaking, the framework developed in this work does not verify the pair approximation as three-body interactions are present in the bending potential. To test indirectly the validity of the pair approximation, we also implemented a version of the anisotropic CG simulations where the intramolecular 1,3 interactions between beads linked by two consecutive bonds is described by a pair potential depending on the distance $R_{\mu o}$ between the two beads and on their relative orientation $\cos \phi_{\mu o}$ and not on the bending angle $\cos \theta_{\mu \nu o}$ as was done in Eq. 35 In this version of the model, the CG potential reads:

$$
\begin{aligned}
W^{\mathrm{CG}} & =\sum_{\mu \nu, \mathrm{nb}} W^{\mathrm{nb}}\left(R_{\mu \nu}, \cos \phi_{\mu \nu}\right)+\sum_{\mu \nu, \text { bond }} W^{\text {bond }}\left(R_{\mu \nu}, \cos \phi_{\mu \nu}\right) \\
& +\sum_{\mu o, 1,3} W^{\text {intra1,3}}\left(R_{\mu o}, \cos \phi_{\mu o}\right)
\end{aligned}
$$

where $\sum_{\mu \nu, \mathrm{nb}}$ and $\sum_{\mu \nu \text {,bond }}$ are sums on pairs of beads, which are non-bonded and directly bonded, respectively, as in Eq. 35 and where $\sum_{\mu o, 1,3}$ denotes the sum on pairs of beads separated by two bonds. The anisotropic CG potential $W^{\text {intra1,3 }}\left(R_{\mu o}, \cos \phi_{\mu o}\right)$ is obtained in the same way as that described in Sec. 3.4 and is displayed in the ESI $\dagger$, Sec. S5 for the level of coarse-graining $\lambda=1$. The non-bonding radial distribution functions obtained with isotropic and anisotropic CG simulations using this potential are compared to their MD counterparts in Fig. 9 for the three levels of coarse-graining. They are also compared to the radial distributions functions obtained in anisotropic CG simulations using the bending potential $W^{\text {bend }}(\cos \theta)$. The difference between each CG result and the corresponding MD result is plotted at the bottom of each figure. Figure 9 contains many pieces of information. The first one is that as in the case of the CG simulations with the bending potential, the isotropic and anisotropic CG simulations using the intramolecular 1,3 pair potential lead to quasi-identical non-bonding radial distribution functions. The reason is also the same (not shown). The second piece of information is that at the coarse-graining level $\lambda=1$ the radial distribution function obtained in CG simulations using the intramolecular 1,3 pair potential $W^{\mathrm{intra1,3}}(R, \cos \phi)$ is further away from its MD counterpart than that obtained in CG simulations using the more classical bending potential $W^{\text {bend }}(\cos \theta)$. However, and this is the third piece of information, as the level of coarse-graining increases this statement is reversed. Surprisingly, the radial distribution function obtained in CG simulations 
using the intramolecular 1,3 pair potential $W^{\text {intra1,3 }}(R, \cos \phi)$ is closer to its MD counterpart before and around the first peak, than that obtained in CG simulations using the bending potential $W^{\text {bend }}(\cos \theta)$. In particular the too large overlap between beads at short distances seen in CG simulations is reduced when the intramolecular 1,3 pair potential $W^{\text {intra1,3 }}(R, \cos \phi)$ is used. The fact that the ability of the CG simulations to reproduce the MD result changes as the level of coarse-graining increases when either the bending potential or the intramolecular 1,3 potential is used is consistent with the fact that none of these potentials is really good. On the first hand, the intramolecular 1,3 potential $W^{\text {intra1,3 }}(R, \cos \phi)$ is a pure pair potential and lacks three-body contributions. On the other hand, the usual bending potential, which has the advantage of incorporating three-body interactions through the bending angle dependence, assumes that the bending angle is independent of the bond lengths for the two bonds involved. There is very probably a coupling between the bond distance and the bending angle as can be considered for example in class II force field 50 and this could be deduced from MD simulations in a bottom-up approach. To conclude, one should say that adding many body terms, in particular intramolecular three-body terms, constitutes a possible way to refine the model without putting into question the general framework developed in Sec. 3 .

\subsection{Main direction of force and effective torque}

To show that the main direction of the force between beads $\mu$ and $\nu$ is indeed the unit vector $\mathbf{e}_{\mu \nu}$ linking the two beads and that the main direction of the effective torque between the two beads is the cross product $\mathbf{u}_{\mu, 1} \times \mathbf{u}_{\nu, 1}$ between the principal directions of the two beads, we compare the force and effective torque projected in these directions to their counterparts projected in a perpendicular direction. A unit vector perpendicular to $\mathbf{e}_{\mu \nu}$ is denoted $\mathbf{e}_{\mu \nu}^{\perp}$ and a unit vector perpendicular to $\mathbf{u}_{\mu, 1} \times \mathbf{u}_{\nu, 1}$ is simply $\mathbf{u}_{\mu, 1}$. The force and torque projected in these different directions are displayed in Fig. 10. As can be seen in Fig. 10 (a), the force projected onto $\mathbf{e}_{\mu \nu}^{\perp}$ is much smaller than onto $\mathbf{e}_{\mu \nu}$ for any value of the distance $R$ and the angle $\cos \phi_{\mu \nu}$, where the force is nonzero. This is not surprising as the isotropic force, depending on the distance alone, has already been shown to be mainly in direction $\mathbf{e}_{\mu \nu}$. Figure 10 (b) shows that the assumption stating that the effective torque is mainly in direction $\mathbf{u}_{\mu, 1} \times \mathbf{u}_{\nu, 1}$ also holds rather well. It is especially true for high values of $\cos \phi_{\mu \nu}$ and small values of $R_{\mu \nu}$ where the effective torque is non-zero. However, this type of test does not account for contributions in the perpendicular direction which would cancel each other on average for the same value of $\cos \phi_{\mu \nu}$ and $R_{\mu \nu}$. This could be the case if the CG potential was better described by the functional form of the Gay-Berne potential which also depends on the angle $\cos \phi_{\mu}=\mathbf{u}_{\mu, 1} \cdot \mathbf{e}_{\mu \nu}$ and $\cos \phi_{\nu}=-\mathbf{u}_{\nu, 1} \cdot \mathbf{e}_{\mu \nu}$. Nevertheless, this layer of assumption is introduced later in the derivation of the CG model compared to the approximations tested successfully in Secs.5.1 to 5.3 and can be easily raised without questioning the general framework of the model. Thus, considering a more refined non-bonding pair potential, depending on more variables,
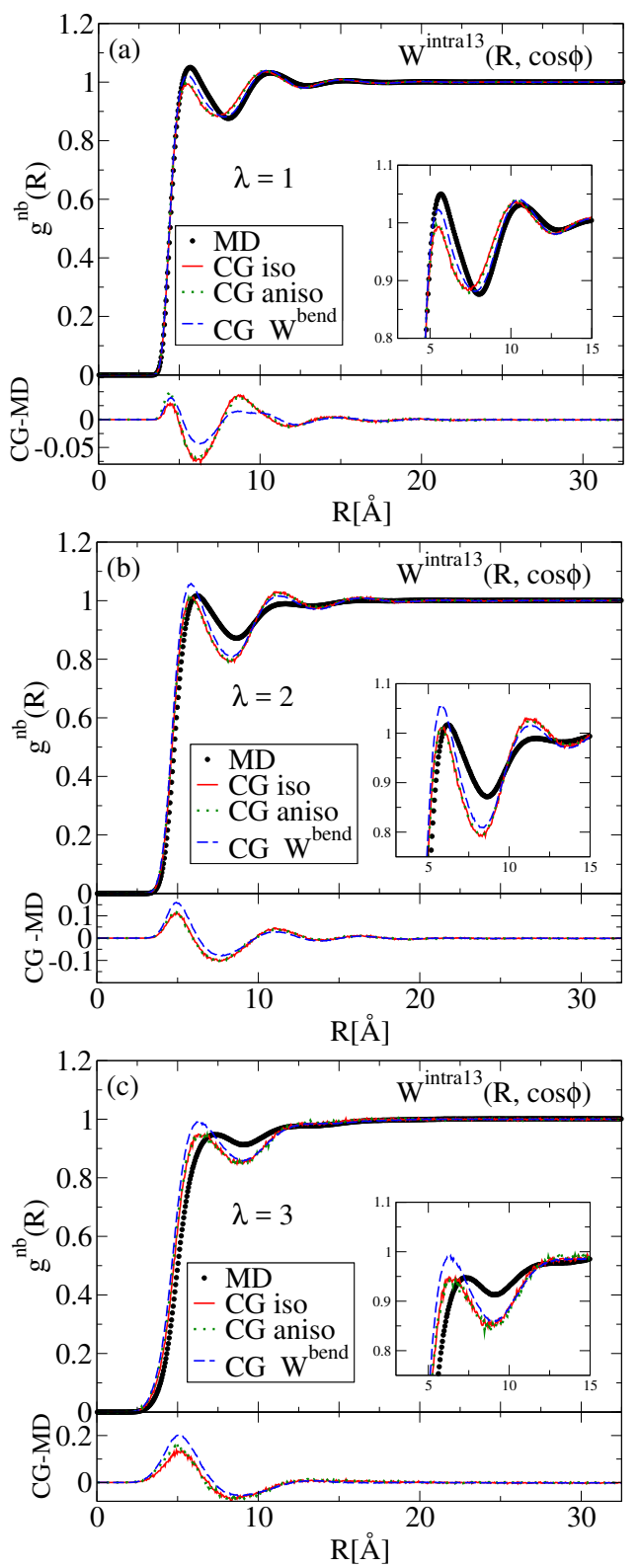

Fig. 9 Same as legend as Fig 7 for CG simulations done with the intramolecular 1,3 pair potential $W^{\text {intra1,3 }}$. 
is another natural perspective of this work.
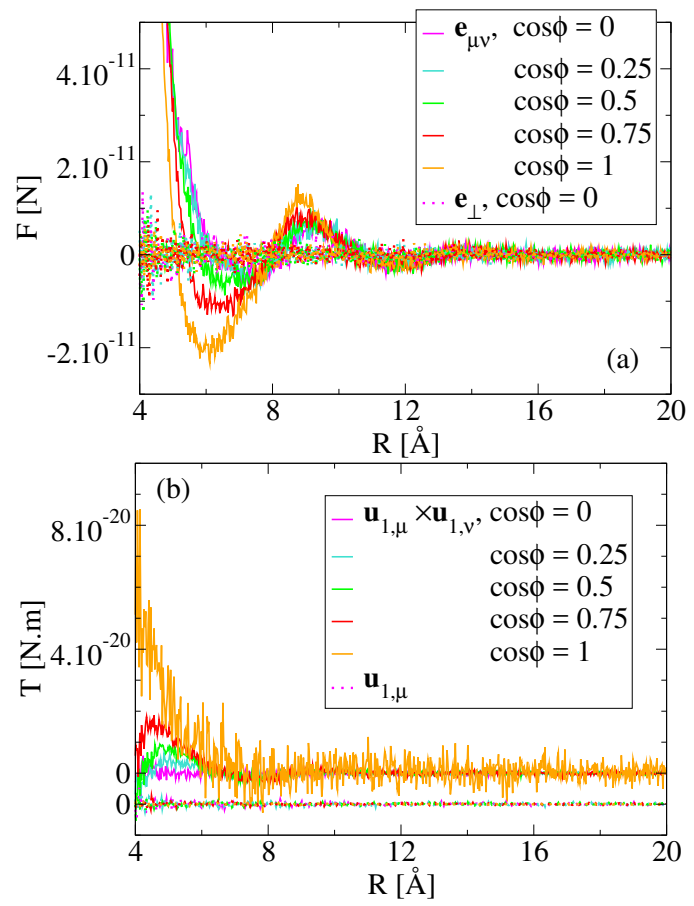

Fig. 10 (a) Variation of the force $\mathbf{F}$ between beads $\mu$ and $\nu$ projected onto $\mathbf{e}_{\mu \nu}$ (solid lines) and $\mathbf{e}_{\mu \nu}^{\perp}$ (dotted lines) with the distance $R$ between the two beads and for different values of the angles $\phi$ between the orientations of the two beads. (b) Same as (a) for the effective torque $\mathbf{T}$ projected onto $\mathbf{u}_{\mu, 1} \times \mathbf{u}_{\nu, 1}$ (solid lines) and $\mathbf{u}_{\mu, 1}$ (dotted lines). To improve readability the effective torque projected onto the perpendicular direction $\mathbf{u}_{\mu, 1}$ has been shifted down but is indeed fluctuating around zero.

\section{Summary and conclusion}

In this work, we developed a model which takes into account the relative orientation between beads in coarse-grained (CG) simulations and derived the corresponding CG potential from atomistic simulations in a bottom-up approach. The CG variables chosen to do so are the usual center of mass position and velocity, but also the quaternion associated to the rotation from the reference basis to the basis of the eigenvectors of the bead inertia tensor and the corresponding angular velocity. As an initial step, the model is developed for conservative forces only, while the derivation of dissipative and random forces will be the subject of a subsequent study. There are two types of results in this work. First, the main assumptions on which the CG model relies are shown to hold quiet well for a cis-1,4-polybutadiene melt. The quaternion and angular velocity of a bead are indeed slow variables in the same sense as the position and velocity of its center of mass while the force and effective torque on a bead are very sensitive to atomic vibrations. The term involving the time derivative of the principal moments of inertia in the equation of motion of the angular velocity is shown to be negligible with respect to other terms in this equation. This reduces the CG equations of motion to rigid-body equations of motion, whose implementation is well documented. Second, the anisotropic CG simulations based on the anisotropic
CG model are able to reproduce well the locally anisotropic structure of the polymer melt. The radial and angular distribution functions obtained in molecular dynamics (MD) and anisotropic CG simulations are globally close at all levels of coarse-graining tested, from 1 to 3 monomers per bead, and for any distance and orientation between the two considered beads.

This work also proposes several directions for improving the anisotropic CG model. Indeed, in its present state the anisotropic CG model predicts the same radial distribution function and same chain dimensions as a more usual isotropic CG model and these are more and more different from their MD counterparts as the level of coarse-graining increases. This is unfortunate and is due to two facts: (i) the anisotropic and isotropic CG pair forces have the exact same direction along the vector linking two centers of mass, (ii) our anisotropic CG simulations reproduce well the conditional probability that two beads have a certain relative orientation given that they are at a specified distance. To increase the agreement between the MD and anisotropic CG structural results, a natural perspective opened by this work is to consider a CG anisotropic potential depending on more terms and leading to perpendicular contributions in the force, like the Gay-Berne potential functional form. This can easily be done while keeping the model general framework. Another direction to improve the structural properties of the anisotropic CG model is to change the intramolecular potential made of the sum of a bonding and bending term into a single three-body intramolecular potential coupling the two bond lengths and the bending angle involved.

Finally, the coarse-graining methodology presented in this work opens the way for the derivation of full anisotropic DPD equations where the conservative, dissipative and random forces can be deduced in a bottom-up approach. Indeed, the chosen CG variables are explicitly given in terms of atomic variables so that the expressions of the memory kernel and random noise given in Ref. ${ }^{-1}$ can in principle be used. This should allow for an accurate calculation of the dynamic and transport properties, after determination of a good friction coefficient for dissipative forces using constrained molecular dynamics simulations ${ }^{4}$. Note, however, that such constrained simulations are not straightforward to implement as the quaternion and angular velocity of a bead are not linear combinations of the atomic positions and velocities.

\section{Conflicts of interest}

There are no conflicts to declare.

\section{Acknowledgements}

The authors would like to acknowledge the SMICE (Simulation de Matériaux pour l'Industrie par Calcul Exaflop) project, which is a PIA (Plan d'Investissement d'Avenir) from the Commissariat Général à l'Investissement.

\section{Notes and references}

1 C. Peter and K. Kremer, Soft Matter, 2009, 5, 04357-4366.

2 W. G. Noid, J. Chem. Phys., 2013, 139, 090901.

3 P. Español and P. B. Warren, J. Chem. Phys., 2017, 146, 150901. 
4 C. Hijón, P. Español, E. Vanden-Eijnden and R. DelgadoBuscalioni, Faraday Discuss., 2010, 144, 301-322.

5 F. Müller-Plathe, ChemPhysChem, 2002, 3, 754-769.

6 D. Reith, M. Pütz and F. Müller-Plathe, J. Comput. Chem., 2003, 24, 1624-1636.

7 F. Ercolessi and J. B. Adams, Eurphus. Lett., 1994, 26, 583588.

8 S. Izvekov, M. Parrinello, C. J. Burnham and G. A. Voth, J. Chem. Phys., 2004, 120, 10896.

9 Z. Li, X. Bian, X. Yang and G. E. Karniadakis, J. Chem. Phys., 2016, 145, 044102.

10 A. Dequidt and J. G. S. Canchaya, J. Chem. Phys., 2015, 143, 084122.

11 K. Kempfer, J. Devémy, A. Dequidt, M. Couty and P. Malfreyt, ACS Omega, 2019, 4, 5955-5967.

12 K. Kempfer, J. Devémy, A. Dequidt, M. Couty and P. Malfreyt, Soft Matter, 2020, 16, 1538-1547.

13 D. Kauzlarić, P. Español, A. Greiner and S. Succi, Macromol. Theory Simul., 2011, 20, 526-540.

14 P. Español and I. Zúñiga, Phys. Chem. Chem. Phys., 2011, 13, 10538-10545.

15 Z. Li, X. Bian, B. Caswell and G. E. Karniadakis, Soft Matter, 2014, 10, 8659.

16 C. A. Lemarchand, M. Couty and B. Rousseau, J. Chem. Phys., 2017, 146, 074904.

17 S. N. Fejer, D. Chakrabarti and D. J. Wales, Soft Matter, 2011, 7, 3553-3564.

18 Z.-W. Li, Y.-L. Zhu, Z.-Y. Lu and Z.-Y. Sun, Soft Matter, 2016, 12, 741-749.

19 M. Deng, W. Pan and G. E. Karniadakis, J. Comput. Phys., 2017, 336, 481-491.

20 Z.-W. Li, Y.-L. Zhu, Z.-Y. Lu and Z.-Y. Sun, Soft Matter, 2018, 14, 7625-7633.

21 N.-V. Buchete, J. E. Straub and D. Thirumalai, J. Chem. Phys., 2003, 118, 7658.

22 N.-V. Buchete, J. E. Straub and D. Thirumalai, Polymer, 2004, 45, 597-608.

23 A. Srivastava and S. Ghosh, Phys. Rev. E, 2012, 85, 026702.

24 T. K. Haxton, R. V. Mannige, R. N. Zuckermann and S. Whitelam, J. Chem. Theory Comput., 2015, 11, 303-315.

25 P. Poier, C. N. Likos, A. J. Moreno and R. Blaak, Macromolecules, 2014, 48, 4983-4997.

26 P. Poier, P. Bacová, A. J. Moreno, C. N. Likos and R. Blaak, Soft Matter, 2016, 12, 4805-4820.

27 K. Kempfer, J. Devémy, A. Dequidt, M. Couty and P. Malfreyt, Macromolecules, 2019, 52, 2736-2747.

28 G. Maurel, B. Schnell, F. Goujon, M. Couty and P. Malfreyt, J. Chem. Theory Comput., 2012, 8, 4570-4579.

29 G. Maurel, F. Goujon, B. Schnell and P. Malfreyt, RSC Adv., 2015, 5, 14065-14073.

30 P. Gao and H. Guo, Polymer, 2015, 69, 25-38.

31 J.-B. Maillet, M. Mareschal, L. Soulard, R. Ravelo, P. S. Lomdahl, T. C. Germann and B. L. Holian, Phys. Rev. E, 2000, 63,
016121.

32 C. A. Lemarchand, D. Bousquet, B. Schnell and N. Pineau, J. Chem. Phys., 2019, 150, 224902.

33 V. A. Harmandaris, M. Doxastakis, V. G. Mavrantzas and D. N. Theodorou, J. Chem. Phys., 2002, 116, 436.

34 G. D. Smith and W. Paul, J. Phys. Chem. A, 1998, 102, 12001208.

35 G. D. Smith, W. Paul, M. Monkenbusch, L. Willner, D. Richter, X. H. Qiu and M. D. Ediger, Macromolecules, 1999, 32, 88578865.

36 S. Melchionna, G. Ciccotti and B. L. Holian, Mol. Phys., 1993, 78, 533-544.

37 P. Zoller and D. Walsh, Standard Pressure-Volume-Temperature Data for Polymers, Technomic Publishing, Lancaster, 1995.

38 P. Gestoso, E. Nicol, M. Doxastakis and D. N. Theodorou, Macromolecules, 2003, 36, 6925-6938.

39 M. P. Allen and D. J. Tildesley, Computer simulation of liquids, Clarendon Press, Oxford, 1989.

40 D. C. Rapaport, J. Comp. Phys., 1985, 60, 306-314.

41 D. J. Cleaver, C. M. Care, M. P. Allen and M. P. Neal, Phys. Rev. E, 1996, 54, 559-567.

42 P. A. Golubkov and P. Ren, The Journal of Chemical Physics, 2006, 125, 064103.

43 M. Allen and G. Germano, Mol. Phys., 2007, 104, 3225-3235.

44 P. M. Rodger, A. Stone and D. Tildesley, Mol. Sim., 1992, 8, 145-164.

45 D. Reith, H. Meyer and F. M uller-Plathe, Macromolecules, 2001, 34, 2335-2345.

46 X. Li, D. Kou, S. Rao and H. Liang, J. Chem. Phys., 2006, 124, 204909.

47 N. Martzel, A. Dequidt, J. Devémy, R. Blaak, S. Garruchet, B. Latour, F. Goujon, E. Munch and P. Malfreyt, Adv. Theory Simul., 2020, 2020, 2000124.

48 M. Babadi, R. Everaers and M. R. Ejtehadi, J. Chem. Phys., 2006, 124, 174708.

49 C. Law, D. J. Ashton, N. B. Wilding and R. L. Jack, J. Chem. Phys., 2016, 145, 084907.

50 H. Sun, J. Phys. Chem. B, 1998, 102, 7338-7364. 